\title{
A GENERALIZED TWO-COMPONENT MODEL OF SOLAR WIND TURBULENCE AND AB INITIO DIFFUSION MEAN-FREE PATHS AND DRIFT LENGTHSCALES OF COSMIC RAYS
}

\author{
T. Wiengarten ${ }^{1}$, S. Oughton ${ }^{2}$, N. E. Engelbrecht ${ }^{3}$, H. Fichtner ${ }^{1}$, J. Kleimann ${ }^{1}$, And K. Scherer $^{1}$ \\ ${ }^{1}$ Institut für Theoretische Physik IV, Ruhr-Universität Bochum, Germany \\ ${ }^{2}$ Department of Mathematics, University of Waikato, Hamilton 3240, New Zealand \\ ${ }^{3}$ Center for Space Research, North-West University, Potchefstroom 2520, South Africa \\ Received 2016 June 12; revised 2016 August 17; accepted 2016 September 2; published 2016 December 2
}

\begin{abstract}
We extend a two-component model for the evolution of fluctuations in the solar wind plasma so that it is fully three-dimensional (3D) and also coupled self-consistently to the large-scale magnetohydrodynamic equations describing the background solar wind. The two classes of fluctuations considered are a high-frequency parallelpropagating wave-like piece and a low-frequency quasi-two-dimensional component. For both components, the nonlinear dynamics is dominanted by quasi-perpendicular spectral cascades of energy. Driving of the fluctuations by, for example, velocity shear and pickup ions is included. Numerical solutions to the new model are obtained using the CRONOS framework, and validated against previous simpler models. Comparing results from the new model with spacecraft measurements, we find improved agreement relative to earlier models that employ prescribed background solar wind fields. Finally, the new results for the wave-like and quasi-twodimensional fluctuations are used to calculate ab initio diffusion mean-free paths and drift lengthscales for the transport of cosmic rays in the turbulent solar wind.
\end{abstract}

Key words: magnetohydrodynamics (MHD) - solar wind - Sun: heliosphere - turbulence

\section{INTRODUCTION}

The explicit consideration and self-consistent implementation of the evolution of turbulence in expanding plasma flows is a focus of contemporary modeling of astrophysical flow phenomena. This is particularly so for the solar wind; see the review-like introductions in Usmanov et al. (2011, 2014), Zank et al. (2012a), and Wiengarten et al. (2015). This considerable improvement, relative to non-self-consistent modeling, is, on the one hand, necessary in order to fully understand the transport of charged energetic particles in the heliosphere (e.g., Engelbrecht \& Burger 2013), and via this to explore the physics of their interactions with the plasma turbulence (e.g., Schlickeiser 2002; Shalchi 2009). On the other hand, the correct description of the transport of cosmic rays in other astrophysical systems is also of great interest. For example, in astrospheres, i.e., circumstellar regions occupied by stellar winds, it is of high relevance in the context of exoplanet research (e.g., Scalo et al. 2007; Grenell et al. 2012; Grießmeier et al. 2015) and potentially for an understanding of cosmic-ray anisotropy at high energy (Scherer et al. 2015). Another example is the, at least partly diffusive, cosmic-ray transport in galactic halos (e.g., Heesen et al. 2009; Mao et al. 2015).

Modeling of the transport of solar wind turbulence has advanced considerably since the early model of $\mathrm{Tu}$ et al. (1984), which was itself a major step forward from WKB transport theory (e.g., Parker 1965; Hollweg 1973). Improved inertial range models (e.g., Marsch \& Tu 1990; Zhou \& Matthaeus 1990) and energy-containing range models (e.g., Matthaeus et al. 1994, 1996; Zank et al. 1996, 2012a) have been presented. These have often included additional effects, such as heating of the solar wind (e.g., Zank et al. 1996; Matthaeus et al. 1999), non-zero cross helicity (e.g., Matthaeus et al. 2004; Breech et al. 2005, 2008), non-constant difference in velocity $\boldsymbol{v}$ and magnetic field $\boldsymbol{b}$ fluctuation energy (sometimes called residual energy, Matthaeus et al. 1994; Zank et al. 2012a; Adhikari et al. 2015), and different correlation lengths for $\boldsymbol{v}$ and $\boldsymbol{b}$ as well as for the Elasser fluctuations (Zank et al. 2012a; Dosch et al. 2013; Adhikari et al. 2015). See Zank et al. (2012a) and Zank (2014) for reviews of this progress.

Another extension concerns the nature of the fluctuations. Models like those mentioned above typically treat the fluctuations as being of a single kind, typically either waves or some form of turbulence. Oughton et al. (2011) developed a model where propagating high-frequency wave-like fluctuations and low-frequency, perpendicularly cascading, thus quasi-two-dimensional (quasi-2D) turbulent fluctuations are both supported (see also Oughton et al. 2006; Isenberg et al. 2010). This approach, referred to as two-component turbulence modeling, explicitly acknowledges the presence of both turbulence and wave-like fluctuations and has distinct advantages compared to the "traditional" one-component modeling. First, it is commonly agreed that there are at least two turbulence drivers, namely stream shear at low frequencies and unstable pickup ion velocity distributions at high frequencies. Clearly, the separation of the turbulence into two corresponding frequency components allows for a more "natural" quantitative formulation and modeling of the distinct driving processes. Second, this decomposition permits a fairly detailed treatment of nonlinear interactions of wave-like and quasi-2D components with each other and among themselves (Oughton et al. 2006, 2011). Third, assuming these two components to determine the slab and 2D turbulence quantities required in contemporary cosmic-ray transport theory with sufficient accuracy, they form the basis of so-called ab initio modeling of cosmic-ray modulation (Engelbrecht \& Burger 2013).

In order to self-consistently couple turbulence transport models to those of the large-scale structure of the heliosphere (e.g., Zank 2015) or astrospheres (e.g., Scherer et al. 2015) the former must be formulated in three spatial dimensions. This has been done for the one-component model by Usmanov et al. (2011). Another generalization concerns the removal of the 
limitation of the model's validity for the super-Alfvénic solar/ stellar wind regimes, which-again for the one-component model-has been achieved recently in a non-self-consistent fashion by Adhikari et al. (2015) and fully self-consistently by Wiengarten et al. (2015). Naturally, it is desirable to make both extensions also for the two-component turbulence model. This is the objective of the present paper, whose structure we now outline.

We formulate the basic equations of the two-component phenomenology and its coupling to the large-scale magnetohydrodynamic (MHD) equations in Section 2. The implementation in the CRONOS numerical framework is presented in Section 3, along with numerical results. These include a computational validation with respect to the simpler Oughton et al. (2011) model, and results from the new two-component model with its more realistic background solar wind. A comparison with spacecraft data is also presented. Then, in Section 4 , the findings are used to calculate diffusion and drift coefficients for the transport of cosmic rays in the heliosphere. We conclude with a summary and an outlook on future improvements in Section 5.

\section{STATEMENT OF THE MODEL AND ITS PHYSICS}

\subsection{Definitions}

We begin by introducing our notation for the large-scale and small-scale fields. The total solar wind velocity is written $\boldsymbol{U}(\boldsymbol{r})+\boldsymbol{v}(\boldsymbol{r}, \boldsymbol{x})$, the sum of a large-scale piece dependent upon the heliocentric position vector $\boldsymbol{r}$, and a small-scale contribution that also depends upon local small-scale coordinates $\boldsymbol{x}$, relative to each $\boldsymbol{r}$. Similarly, the total magnetic field is $\boldsymbol{B}(\boldsymbol{r})+\boldsymbol{b}(\boldsymbol{r}, \boldsymbol{x})$, with associated large-scale Alfvén speed $\boldsymbol{V}_{\mathrm{A}}=\boldsymbol{B} / \sqrt{4 \pi \rho}$, where $\rho(\boldsymbol{r})$ is the large-scale mass density. The small-scale dynamics is treated as incompressible (see Zank et al. 2012b for a discussion of transport of density fluctuations). As a simplifying assumption, the fluctuation amplitudes, $\boldsymbol{v}$ and $\boldsymbol{b}$, are restricted to be transverse to $\boldsymbol{B}$; that is, parallel variances are neglected. Solar wind observations indicate that this is often a reasonable approximation (e.g., Belcher \& Davis 1971; Klein et al. 1991). In general, the above quantities are also time-dependent.

The large-scale wind velocity $\boldsymbol{U}$ is with respect to an inertial frame; in the frame corotating with the Sun the large-scale velocity is $\boldsymbol{V}=\boldsymbol{U}-\boldsymbol{\Omega} \times \boldsymbol{r}$, where $\boldsymbol{\Omega}$ is the solar angular rotation rate. Our numerical computations are often performed in this corotating frame. In obtaining the transport equations in this frame, we make use of the relation $\nabla \cdot \boldsymbol{V}=\nabla \cdot \boldsymbol{U}$, which holds because $\nabla \cdot(\boldsymbol{\Omega} \times \boldsymbol{r})=0$.

The two-component aspect of the model involves separating the fluctuations into two precisely defined incompressible elements: quasi-2D turbulence and a complementary wave-like component (Oughton et al. 2006, 2011). Specifically, employing Elasser variables, $\boldsymbol{z}^{ \pm}=\boldsymbol{v} \pm \boldsymbol{b} / \sqrt{4 \pi \rho(\boldsymbol{r})}$, we express the fluctuations as

$$
\boldsymbol{z}^{ \pm}(\boldsymbol{r}, \boldsymbol{x})=\boldsymbol{q}^{ \pm}+\boldsymbol{w}^{ \pm}
$$

where $\boldsymbol{q}^{ \pm}$and $\boldsymbol{w}^{ \pm}$are the quasi-2D and wave-like components, respectively; both quantities are functions of the (large-scale) heliocentric radius $\boldsymbol{r}$ and the small-scale displacements $\boldsymbol{x}$ from each $\boldsymbol{r}$.

Table 1 summarizes the definitions of the major energyrelated fluctuation quantities, which appear in the transport
Table 1

Definitions of Some Important Physical Variables for the Quasi-2D and Wave-like Components

\begin{tabular}{llc}
\hline \hline $\begin{array}{l}\text { Quasi-2D } \\
\text { Fluctuations }\end{array}$ & Quantity & $\begin{array}{c}\text { Wave-like } \\
\text { Fluctuations }\end{array}$ \\
\hline$Z_{ \pm}^{2}=\left\langle\boldsymbol{q}_{ \pm} \cdot \boldsymbol{q}_{ \pm}\right\rangle$ & Elasser "energies" & $W_{ \pm}^{2}=\left\langle\boldsymbol{w}_{ \pm} \cdot \boldsymbol{w}_{ \pm}\right\rangle$ \\
$2 Z^{2}=Z_{+}^{2}+Z_{-}^{2}$ & total "energies" & $2 W^{2}=W_{+}^{2}+W_{-}^{2}$ \\
$2 H_{c}^{z}=Z_{+}^{2}-Z_{-}^{2}$ & cross helicities & $2 H_{c}^{w}=W_{+}^{2}-W_{-}^{2}$ \\
$\sigma_{c, z}=\frac{Z_{+}^{2}-Z_{-}^{2}}{Z_{+}^{2}+Z_{-}^{2}}$ & normalized cross helicities & $\sigma_{c, w}=\frac{W_{+}^{2}-W_{-}^{2}}{W_{+}^{2}+W_{-}^{2}}$ \\
$\sigma_{D}^{z}=\frac{\left\langle\boldsymbol{q}_{+} \cdot \boldsymbol{q}_{-}\right\rangle}{Z^{2}}$ & normalized energy differences & $\sigma_{D}^{w}=\frac{\left\langle\boldsymbol{w}_{+} \cdot \boldsymbol{w}_{-}\right\rangle}{W^{2}}$
\end{tabular}

Note. Angle brackets $\langle\cdots\rangle$ indicate averaging over the small-scale coordinate $\boldsymbol{x}$ (at each large-scale coordinate $\boldsymbol{r}$ ). Note that $H_{c}^{z}$ and $H_{c}^{w}$ differ by a factor of two from the definitions used in Oughton et al. (2011).

model. For the quasi-2D component, $\sigma_{c, z}$ is the normalized cross helicity, and $\sigma_{D}^{z}$ the normalized energy difference, equal to the (normalized) kinetic energy less the magnetic energy all divided by the sum of these. In general, the analogous quantity for the wave-like component is indicated by a subscript or superscript $w$.

Along with the energies (per mass) of the fluctuations, $Z_{ \pm}^{2}$ and $W_{ \pm}^{2}$, it is also necessary to consider their characteristic lengthscales, typically defined using correlation lengths. In general, these are distinct for each type of field; for example, $\ell_{+}$ for $Z_{+}^{2}$ and $\ell_{-}$fo $Z_{-}^{2}$. Here we make the simplifying assumption that these scales are equal and denote the characteristic lengthscale of $Z^{2}$ as $\ell$ and that of $W^{2}$ as $\lambda$. In addition, the typical parallel scale of the wave-like component, $\lambda_{\|}$, is needed, particularly in connection with driving by pickup ions. (For one-component transport models that consider the \pm lengthscales separately see Zank et al. 2012a and Adhikari et al. 2015.)

Finally, in this section, we address the suitability of using incompressible MHD to model solar wind fluctuations. Naturally, the actual solar wind fluctuations will often display some compressive activity. Here, however, from the outset we approximate them as being incompressible and thus neglect small-scale compressive behavior. On the observational side, density fluctuations are often found to be $\sim 10 \%$ of the mean value (e.g., Roberts et al. 1987; Matthaeus et al. 1991), providing motivation for neglecting compressive activity at this level. On the theory side, the nearly incompressible (NI) approach for systems with small Mach numbers (Zank \& Matthaeus 1992, 1993), leads to a leadingorder description that is either incompressible 3D MHD (large plasma beta) or incompressible 2D MHD (beta small or order unity). The next order corrections are termed "NI" and support MHD waves. In particular, when beta is of the order of unity, as is typical for the solar wind, the NI solutions include Alfvén waves with timescales shorter than those associated with the leading-order incompressible behavior. Thus, modeling the system as we do herein, i.e., using incompressible quasi-2D and incompressible wave-like components, is consistent with the NI results.

\subsection{The Transport Model for the Fluctuations}

The transport and driving terms-for the energy, cross helicity, and characteristic lengthscales of the fluctuations- 
have been derived and discussed in various works (e.g., Matthaeus et al. 1994; Usmanov et al. 2011; Zank et al. 2012a). Here we largely follow the approach of Matthaeus et al. (1994) and Usmanov et al. (2011), extended to incorporate the homogeneous two-component phenomenology presented in Oughton et al. (2011) and also retaining terms of the order of $V_{\mathrm{A}} / U$ (Adhikari et al. 2015; Wiengarten et al. 2015).

This leads to the following equations for the fluctuation energies, in the frame corotating with the Sun,

$$
\begin{aligned}
\frac{\partial Z^{2}}{\partial t}= & -\nabla \cdot\left(\boldsymbol{V} Z^{2}+\boldsymbol{V}_{\mathrm{A}} H_{c}^{z}\right)+2 \boldsymbol{V}_{\mathrm{A}} \cdot \nabla H_{c}^{z}+\frac{1}{2}(\nabla \cdot \boldsymbol{U}) Z^{2} \\
& -\sigma_{D}^{z} Z^{2}\left[\frac{\nabla \cdot \boldsymbol{U}}{2}-\hat{\boldsymbol{B}} \cdot(\hat{\boldsymbol{B}} \cdot \nabla) \boldsymbol{U}\right] \\
& -\alpha_{z}\left[\frac{Z^{3}}{\ell} f_{z z}^{+}+\frac{2 W Z^{2}}{\ell} \frac{f_{z w}^{+}}{1+Z / W}\right]+\alpha_{z} X^{+} \\
& +\frac{Z^{2}}{r} C_{\mathrm{sh}}^{Z}|\boldsymbol{U}|,
\end{aligned}
$$

$$
\begin{aligned}
\frac{\partial W^{2}}{\partial t}= & -\nabla \cdot\left(\boldsymbol{V} W^{2}+\boldsymbol{V}_{\mathrm{A}} H_{c}^{w}\right)+2 \boldsymbol{V}_{\mathrm{A}} \cdot \nabla H_{c}^{w}+\frac{1}{2}(\nabla \cdot \boldsymbol{U}) W^{2} \\
& -\sigma_{D}^{w} W^{2}\left[\frac{\nabla \cdot \boldsymbol{U}}{2}-\hat{\boldsymbol{B}} \cdot(\hat{\boldsymbol{B}} \cdot \nabla) \boldsymbol{U}\right] \\
& -\alpha_{w}\left[\frac{2 W^{2} Z}{\lambda} \frac{f_{w z}^{+}}{1+\lambda / \ell}+\frac{2 W^{4} \lambda_{\|}}{\lambda^{2} V_{\mathrm{A}}}\left(1-\sigma_{c, w}^{2}\right)\right]-\alpha_{z} X^{+} \\
& +\frac{W^{2}}{r} C_{\mathrm{sh}}^{W}|\boldsymbol{U}|+\dot{E}_{\mathrm{PI}},
\end{aligned}
$$

$$
\begin{aligned}
\frac{\partial H_{c}^{z}}{\partial t}= & -\nabla \cdot\left(\boldsymbol{V} H_{c}^{z}+\boldsymbol{V}_{\mathrm{A}} Z^{2}\right)+2 \boldsymbol{V}_{\mathrm{A}} \cdot \nabla Z^{2}+\frac{1}{2}(\nabla \cdot \boldsymbol{U}) H_{c}^{z} \\
& +\sigma_{D}^{z} Z^{2}\left[\nabla \cdot \boldsymbol{V}_{\mathrm{A}}+\frac{\hat{\boldsymbol{B}} \cdot(\hat{\boldsymbol{B}} \cdot \nabla) \boldsymbol{B}}{\sqrt{4 \pi \rho}}\right] \\
& -\alpha_{z}\left[\frac{Z^{3}}{\ell} f_{z z}^{-}+\frac{2 W Z^{2}}{\ell} \frac{f_{z w}^{-}}{1+Z / W}\right]+\alpha_{z} X^{-},
\end{aligned}
$$

$$
\begin{aligned}
\frac{\partial H_{c}^{w}}{\partial t}= & -\nabla \cdot\left(\boldsymbol{V} H_{c}^{w}+\boldsymbol{V}_{\mathrm{A}} W^{2}\right)+2 \boldsymbol{V}_{\mathrm{A}} \cdot \nabla W^{2}+\frac{1}{2}(\nabla \cdot \boldsymbol{U}) H_{c}^{w} \\
& +\sigma_{D}^{w} W^{2}\left[\nabla \cdot \boldsymbol{V}_{\mathrm{A}}+\frac{\hat{\boldsymbol{B}} \cdot(\hat{\boldsymbol{B}} \cdot \nabla) \boldsymbol{B}}{\sqrt{4 \pi \rho}}\right] \\
& -\alpha_{w}\left[\frac{2 W^{2} Z}{\lambda} \frac{f_{w z}^{-}}{1+\lambda / \ell}\right]-\alpha_{z} X^{-},
\end{aligned}
$$

where

$$
\begin{gathered}
X^{ \pm}=Y^{+} \pm Y^{-}, \\
Y^{ \pm}=W_{ \pm} Z_{ \pm}\left[\frac{Z_{\mp}}{\lambda} \Gamma_{w}^{z_{\mp} w_{ \pm}}+\frac{W_{\mp}}{\lambda} \Gamma_{w}^{w_{\mp} w_{ \pm}}\right. \\
\left.-\frac{Z_{\mp}}{\ell} \Gamma_{w}^{z_{\mp} z_{ \pm}}-\frac{W_{\mp}}{\ell} \Gamma_{w}^{w_{\mp} z_{ \pm}}\right],
\end{gathered}
$$

$$
\begin{gathered}
\Gamma_{c}^{a b}=\frac{1}{1+\tau_{\mathrm{nl}}^{a b} / \tau_{\mathrm{A}}^{c}}, \\
2 f_{a b}^{ \pm}=\left(1+\sigma^{a}\right) \sqrt{1-\sigma^{b}} \pm\left(1-\sigma^{a}\right) \sqrt{1+\sigma^{b}},
\end{gathered}
$$

with $\sigma^{a} \equiv \sigma_{c, z}$ or $\sigma_{c, w}$ for the component $a=Z$ or $W$. Equation (9) defines various ' $f$ ' functions, bounded by \pm 1 . These act as attenuation factors for the modeled nonlinear terms when the cross helicities are non-zero, as is appropriate (see, e.g., Dobrowolny et al. 1980a).

The $Y^{+}$term, which may be positive or negative, models the exchange of excitation between $Z_{+}^{2}$ and $W_{+}^{2}$, and similarly for $Y^{-}$. The $\Gamma_{c}^{a b}$ are associated with the decay rate of the triple correlation for the term being modeled, and involve the nonlinear $\left(\tau_{\mathrm{nl}}\right)$ and Alfvén $\left(\tau_{\mathrm{A}}\right)$ timescales of the appropriate components. Further details are given in Oughton et al. (2006).

Structurally, we have written Equations (2)-(5) so that different types of physics appear on separate lines. On the first lines, we have advection, expansion, and propagation effects (essentially the WKB terms). The "mixing" terms, proportional to a $\sigma_{D}$ (Zhou \& Matthaeus 1990), are on the second lines. The third line in each equation presents the homogeneous decay phenomenology terms. If there is any forcing, the terms modeling those effects appear as a fourth line. For example, the quasi-2D and wave-like energies are driven by large-scale velocity shear-modeled using either self-consistently computed velocity gradients (Wiengarten et al. 2015) or ad hoc terms in the manner of earlier models (e.g., Zank et al. 1996; Breech et al. 2008) - and $W^{2}$ is also forced by waves generated during the near isotropization of pickup ions $\left(\dot{E}_{\mathrm{PI}}\right)$.

Note that the right-most mixing terms in Equations (4) and (5) are absent from the model of Zank et al. (2012a) on setting their suggested structural similarity parameters for axisymmetric quasi-2D fluctuations, namely $a=1 / 2, b=0$. We find, however, that in order to recover the model of Matthaeus et al. (1994) it is appropriate to choose $a=b=1 / 2$.

Since in each of the Equations (2)-(5) the final line arises from a turbulence phenomenology (Oughton et al. 2011), the terms on these lines are only determined to within $O(1)$ multiplying constants. There are some constraints on these constants; for example, when adding the $Z^{2}$ and $W^{2}$ equations, we require that the exchange terms cancel. Here, we adopt the simplest approach of using a single constant in each equation (except for the variations required in connection with the exchange terms), denoted $\alpha_{z}$ and $\alpha_{w}$.

Transport equations for the characteristic lengthscales- $\ell, \lambda$, $\lambda_{\|}$-are derived following the approach of Matthaeus et al. (1994). This is based on integrating correlation functions over the (small-scale) lag, $\boldsymbol{\xi}$. For example, in the case of $\ell$, one starts with transport equations for $R^{ \pm}(\boldsymbol{r}, \boldsymbol{\xi})=\left\langle\boldsymbol{q}^{ \pm}(\boldsymbol{r}, \boldsymbol{x}) \cdot \boldsymbol{q}^{ \pm}(\boldsymbol{r}, \boldsymbol{x}+\boldsymbol{\xi})\right\rangle$, defines $L_{ \pm}=\int_{0}^{\infty} R^{ \pm}(\boldsymbol{r}, \boldsymbol{\xi}) \mathrm{d} \xi$ and obtains their transport equations, adds these to give an equation for $L=L_{+}+L_{-}=2 Z^{2} \ell$, and then extracts the equation for $\ell$. The choice of integration direction, $\hat{\boldsymbol{\xi}}$, is discussed below. (See Zank et al. 2012a for a distinct approach.) With the extension to 
two components and retention of $O\left(V_{\mathrm{A}}\right)$ terms, this leads to

$$
\begin{aligned}
\frac{\partial \ell}{\partial t}= & -\boldsymbol{V} \cdot \nabla \ell+\sigma_{c, z} \boldsymbol{V}_{\mathrm{A}} \cdot \nabla \ell \\
& +\frac{L_{D}}{Z^{2}}\left[\nabla \cdot \frac{\boldsymbol{U}}{2}-2 \hat{\xi}_{i} \hat{\xi}_{j} \frac{\partial U_{i}}{\partial r_{j}}\right] \\
& +\ell \sigma_{D}^{z}\left[\nabla \cdot \frac{\boldsymbol{U}}{2}-\hat{\boldsymbol{B}} \cdot(\hat{\boldsymbol{B}} \cdot \nabla) \boldsymbol{U}\right] \\
& +\beta_{z}\left[f_{z z}^{+} Z+f_{z w}^{+} \frac{2 W}{1+Z / W}-\frac{\ell X^{+}}{Z^{2}}\right], \\
\frac{\partial \lambda}{\partial t}= & -\boldsymbol{V} \cdot \nabla \lambda+\sigma_{c, w} \boldsymbol{V}_{\mathrm{A}} \cdot \nabla \lambda \\
& +\frac{\tilde{L}_{D}}{W^{2}}\left[\nabla \cdot \frac{\boldsymbol{U}}{2}-2 \hat{\xi}_{i} \hat{\xi}_{j} \frac{\partial U_{i}}{\partial r_{j}}\right] \\
& +\lambda \sigma_{D}^{w}\left[\nabla \cdot \frac{\boldsymbol{U}}{2}-\hat{\boldsymbol{B}}^{2} \cdot(\hat{\boldsymbol{B}} \cdot \nabla) \boldsymbol{U}\right] \\
& +\beta_{w}\left[\frac{2 f_{w z}^{+} Z}{1+\lambda / \ell}+2\left(1-\sigma_{c, w}^{2}\right) \frac{W^{2} \lambda_{\|}}{\lambda V_{\mathrm{A}}}+\frac{\alpha_{z} \lambda X^{+}}{\alpha_{w} W^{2}}\right],
\end{aligned}
$$

$$
\begin{aligned}
\frac{\partial \lambda_{\|}}{\partial t}= & -\boldsymbol{V} \cdot \nabla \lambda_{\|}+\sigma_{c, w} \boldsymbol{V}_{\mathrm{A}} \cdot \nabla \lambda_{\|} \\
& +0 \quad(\text { mixing terms cancel }) \\
& +2 \alpha_{w}\left(1-\sigma_{c, w}^{2}\right) \frac{W^{2} \lambda_{\|}}{V_{\mathrm{A}} \lambda^{2}} \lambda_{\|} \\
& -\left(\lambda_{\|}-\lambda_{\text {res }}\right) \frac{\dot{E}_{\mathrm{PI}}}{W^{2}}
\end{aligned}
$$

Again the presentation structure has advection, expansion, and wave propagation terms on the first lines, mixing terms (when nonzero) on the second and third lines, turbulence phenomenology terms on the line after those, and any forcing on a separate line after that. In the general case, terms associated with shear driving also appear in the lengthscale equations (e.g., Matthaeus et al. 1996; Zank et al. 1996, 2012a; Breech et al. 2008; Oughton et al. 2011). Herein, however, we assume that shear driving occurs at the correlation scales and thus $\ell, \lambda$, and $\lambda_{\|}$are unaffected by such forcings.

Because $\ell$ and $\lambda$ are characteristic transverse lengthscales, in Equations (10) and (11), the unit vector $\hat{\boldsymbol{\xi}}$ must be chosen to lie in the plane perpendicular to $\boldsymbol{B}$, i.e., in the plane of the fluctuation amplitudes. For a $\boldsymbol{B}$ that lies in the $R-T$ plane, such as the Parker spiral field, a useful choice is $\hat{\boldsymbol{\xi}}=\hat{\boldsymbol{\vartheta}}$, where $\vartheta$ is the polar angle in heliocentric spherical coordinates. (See Matthaeus et al. 1994, where $\hat{\boldsymbol{\xi}}$ is denoted as $\hat{\boldsymbol{r}}$.)

In general, one also needs equations for the energy difference lengthscales (Matthaeus et al. 1994; Zank et al. 2012a; Adhikari et al. 2015). Here we employ the closures $L_{D}=\ell \sigma_{D}^{z} Z^{2}$ and $\tilde{L}_{D}=\lambda \sigma_{D}^{w} W^{2}$. These imply equality of the correlation lengths for the velocity and magnetic fields $\left(\ell_{v}=\ell_{b} ; \quad \lambda_{v}=\lambda_{b}\right)$, and induce slight simplifications of Equations (10) and (11).

In obtaining the equation for $\lambda_{\|}$, we assume that the correlation functions for the $W$ component have the same symmetry structure as that for "slab" Alfvén waves and integrate along the mean field direction: $\hat{\boldsymbol{\xi}}=\hat{\boldsymbol{B}}$. We also make the approximation of a single parallel lengthscale, e.g., $\lambda_{\|, D}=\lambda_{\|}$. These features combine to cause cancellation of the mixing terms. The energy injection associated with (near) isotropization of pickup-ion-induced waves occurs at the gyroradius of the pickup protons, $\lambda_{\text {res }}(\boldsymbol{r})=2 \pi U(\boldsymbol{r}) / \Omega_{\mathrm{p}}(\boldsymbol{r})$ with the proton gyrofrequency $\Omega$.

To close the model, assuming that the large-scale fields like $\boldsymbol{V}$ and $\boldsymbol{V}_{\mathrm{A}}$ are known, we require knowledge of the normalized energy differences, $\sigma_{D}^{z}, \sigma_{D}^{w}$. Their transport equations are obtained in a similar fashion to the above derivations (Matthaeus et al. 1994; Zank et al. 2012a; Adhikari et al. 2015). Herein, however, we approximate $\sigma_{D}^{z}$ and $\sigma_{D}^{w}$ as constant parameters, on the basis of rough observational support (Roberts et al. 1987; Perri \& Balogh 2010; Iovieno et al. 2016). This yields a closed set of equations for the fluctuations, given the large-scale fields. Transport equations for the latter are now considered.

\subsection{Large-scale Equations}

The fluctuations in the present model consist of two different components. This leads to some modified terms in the largescale momentum equation. The single fluctuation component form is given in Usmanov et al. (2011), see their Equation (B2), as

$$
\begin{aligned}
& \frac{\partial(\rho \boldsymbol{U})}{\partial t}+\nabla \cdot\left[\rho \boldsymbol{V} \boldsymbol{U}-\frac{\eta}{4 \pi} \boldsymbol{B} \boldsymbol{B}+\left(P+\frac{B^{2}}{8 \pi}+p_{\text {fluct }}\right) \boldsymbol{I}\right] \\
& \quad=-\rho(\boldsymbol{g}+\boldsymbol{\Omega} \times \boldsymbol{U})
\end{aligned}
$$

where $\Omega=\Omega \boldsymbol{e}_{z} ; \Omega=14.71 \mathrm{day}^{-1}$ (Snodgrass \& Ulrich 1990), and $\boldsymbol{g}=\left(G M_{\odot} / r^{2}\right) \boldsymbol{e}_{r}$ describes the Sun's gravitational acceleration, and $P$ is the large-scale gas pressure.

The forms of $\eta$ and the pressure of the fluctuations $p_{\text {fluct }}$ depend upon the assumed symmetries of the latter, e.g., via the modeling of the MHD Reynolds stress (Usmanov et al. 2011). For the present (transverse, axisymmetric) two-component case, they become

$$
\begin{gathered}
\eta^{2 \mathrm{cpt}}=1+\frac{\sigma_{D}^{z} Z^{2}+\sigma_{D}^{w} W^{2}}{2 V_{\mathrm{A}}^{2}}, \\
p_{\text {fluct }}^{2 \mathrm{cpt}}=\left(1+\sigma_{D}^{z}\right) \frac{\rho Z^{2}}{4}+\left(1+\sigma_{D}^{w}\right) \frac{\rho W^{2}}{4},
\end{gathered}
$$

and are used in place of $\eta$ and $p_{\text {fluct }}$ in Equation (13), which is otherwise unchanged. "Cross-component" effects like $\left\langle\boldsymbol{b}_{Z} \boldsymbol{b}_{W}\right\rangle$ with $\boldsymbol{b}=\boldsymbol{b}_{Z}+\boldsymbol{b}_{W}$ have been neglected. Note that (15) is equivalent to the kinetic (not magnetic) pressure of the fluctuations, though this is a little misleading (physically) since the term is actually the sum of the fluctuation magnetic pressure and contributions from modeling of the MHD Reynolds stresses (Usmanov et al. 2011).

An equation for the total energy density is straightforward to obtain (e.g., Usmanov et al. 2011). However, due to a feature of the CRONOS code, we work instead with the energy density associated with unforced ideal MHD,

$$
e=\frac{\rho U^{2}}{2}+\frac{B^{2}}{8 \pi}+\frac{P}{\gamma-1},
$$


where the full energy density also includes gravitational potential energy and the turbulence energy, $\rho\left(Z^{2}+W^{2}\right) / 2$. These "missing" terms in $e$ are accounted for using source terms in the energy equation (Wiengarten et al. 2015, Appendix B). Following the latter approach with a $\gamma=5 / 3$ adiabatic equation of state and Hollweg's heat flux $\boldsymbol{q}_{H}$ (Hollweg 1974, 1976) yields

$$
\begin{aligned}
\partial_{t} e+ & \nabla \cdot\left[e \boldsymbol{V}+\left(P+\frac{|\boldsymbol{B}|^{2}}{8 \pi}\right) \boldsymbol{U}-(\boldsymbol{U} \cdot \boldsymbol{B}) \frac{\boldsymbol{B}}{4 \pi}\right. \\
- & \left.\boldsymbol{V}_{\mathrm{A}} \rho \frac{H_{c}}{2}+\boldsymbol{q}_{H}\right] \\
= & -\rho \boldsymbol{V} \cdot \boldsymbol{g}-\boldsymbol{U} \cdot \nabla p_{\mathrm{fluct}}^{2 \mathrm{cpt}} \\
& -\frac{H_{c}}{2} \boldsymbol{V}_{\mathrm{A}} \cdot \nabla \rho-\rho \boldsymbol{V}_{\mathrm{A}} \cdot \nabla H_{c} \\
& +\boldsymbol{U} \cdot(\boldsymbol{B} \cdot \nabla)\left[\left(\eta^{2 \mathrm{cpt}}-1\right) \frac{\boldsymbol{B}}{4 \pi}\right] \\
& +\rho\left[\alpha_{z}\left(\frac{f_{z z}^{+} Z^{3}}{2 \ell}+\frac{f_{z w}^{+}}{1+Z / W} \frac{W Z^{2}}{\ell}\right)\right. \\
& \left.+\alpha_{w}\left(\frac{f_{w z}^{+}}{1+\lambda / \ell} \frac{Z W^{2}}{\lambda}+\left(1-\sigma_{c, w}^{2}\right) \frac{W^{4} \lambda_{\|}}{\lambda^{2} V_{\mathrm{A}}}\right)\right],
\end{aligned}
$$

where $H_{c}=H_{c}^{z}+H_{c}^{w}$.

The equations describing the evolution of the large-scale density and magnetic field are unaffected by the extension to incompressible two-component fluctuations. Neglecting the turbulent electric field, one has (e.g., Usmanov et al. 2011; Wiengarten et al. 2015),

$$
\begin{gathered}
\partial_{t} \rho+\nabla \cdot(\rho \boldsymbol{V})=0, \\
\partial_{t} \boldsymbol{B}+\nabla \cdot(\boldsymbol{V} \boldsymbol{B}-\boldsymbol{B} \boldsymbol{V})=\mathbf{0} .
\end{gathered}
$$

\section{NUMERICAL RESULTS}

We use the numerical MHD framework CRONOS to implement the two-component phenomenology of turbulence transport described in the previous section (Equations (2)-(5) and (10)-(12)) and the partner large-scale MHD Equations (13) and (17)-(19). A detailed description of the code's features is available in Wiengarten et al. (2015). In Section 3.1, we present a validation study that compares our new, generalized twocomponent model with the earlier one by Oughton et al. (2011), that prescribed all large-scale fields. Section 3.2 discusses results from the full model, which includes a more realistic background solar wind.

\subsection{Validation}

In order to validate the implementation in CRONOS, we compare results obtained in Oughton et al. (2011) with those from an appropriately restricted form of the new model's equations. Specifically, the background solar wind is prescribed to be a uniform and constant radial flow with $\boldsymbol{U}=440 \mathrm{~km} \mathrm{~s}^{-1} \boldsymbol{e}_{r}$, and a proton number density profile $n=n_{0}\left(r_{0} / r\right)^{2}$ where $n_{0}=n\left(r_{0}=0.3 \mathrm{au}\right)=66 \mathrm{~cm}^{-3}$. The large-scale magnetic field is a Parker spiral, expressed in terms of a vector potential (e.g., Wiengarten et al. 2015),

$$
\boldsymbol{A}=-B_{0} r_{0}^{2} \sin (\vartheta)\left(\frac{\varphi}{r}+\frac{\Omega}{U}\right) \boldsymbol{e}_{\vartheta},
$$

where $\vartheta$ and $\varphi$ are the polar and azimuthal angles in (heliocentric) spherical polar coordinates and $B_{0}=43 \mathrm{nT}$. Additionally, the turbulence transport equations are relieved of all advection and mixing terms involving the Alfvén velocity (but retain the dissipation and interchange terms), as well as the advection and mixing terms in the lengthscale equations. The energy density equation, (17), simplifies considerably and can be usefully re-expressed via $P=2 n k T$ in Equation (16) in terms of the proton temperature (Oughton et al. 2011, Equation (14)); in the present study, however, it is the energy density equation that is solved. The equations are then formally equivalent to those of Oughton et al. (2011), where the sources of turbulence considered are stream shear (modeling the influence of, e.g., corotating interaction regions) and isotropization of pickup ion distributions. While the stream shear drives both the quasi-2D and the wave-like component (so that $C_{\mathrm{sh}}^{Z, W}=1$, see below), the pickup-ion driving feeds the wavelike component only and is approximated as (Zank et al. 1996; Williams et al. 1997)

$$
\dot{E}_{\mathrm{PI}}=\frac{\zeta U^{2} n_{H}}{n_{\mathrm{sw}} \tau_{\text {ion }}} \exp \left(-\frac{L_{\mathrm{cav}}}{r} \frac{\Psi}{\sin (\Psi)}\right)
$$

where $n_{H}=0.1 \mathrm{~cm}^{-3}$ is the interstellar neutral hydrogen density, $\tau_{\text {ion }}=1.33 \times 10^{6} \mathrm{~s}$ is the hydrogen ionization time at $1 \mathrm{au}, L_{\mathrm{cav}}=5.6 \mathrm{au}$ is the characteristic scale of the ionization cavity of the Sun, and $n_{\mathrm{sw}}=6 \mathrm{~cm}^{-3}$ is the solar wind density at $1 \mathrm{au}$. The angle $\Psi$ is that between the observation point and the upwind direction; for pickup ions entering the heliosphere along the $x$-axis, it corresponds to heliospheric latitude, so that above the poles the effective ionization cavity is larger by a factor of $\pi / 2$, and this pushes the region where pickup-ion heating is important to larger $r$. The factor $\zeta$ describes the fraction of the available energy actually channeled into the fluctuations and is mainly a function of the ratio of Alfvén speed to solar wind speed according to the model of Isenberg et al. (2003) and Isenberg (2005) that is used in Section 3.2. For this validation case, we assume a constant $\zeta=0.04$. The Kármán-Taylor constants are set as $\alpha_{z}=\alpha_{w}=2 \beta_{z}=2 \beta_{w}=0.25$ and the residual energies are assumed constant with $\sigma_{D}^{z}=\sigma_{D}^{w}=-1 / 3$ (e.g., Roberts et al. 1987; Perri \& Balogh 2010).

The computational domain extends from 0.3 to $100 \mathrm{au}$ and is covered with 300 cells of increasing cell size $\Delta r$ from 10 to 250 solar radii, while azimuthal symmetry is assumed and the computations are restricted to the ecliptic plane. The remaining inner boundary values at $r_{0}=0.3$ au are $Z^{2}=1500 \mathrm{~km}^{2} \mathrm{~s}^{-2}$, $W^{2}=150 \quad \mathrm{~km}^{2} \mathrm{~s}^{-2}, \quad \sigma_{c, z}=\sigma_{c, w}=0.6, \quad l=\lambda=0.008 \mathrm{au}$, $\lambda_{\|}=0.036 \mathrm{au}$ and $T=1.6 \times 10^{5} \mathrm{~K}$.

Figure 1 shows the resulting behavior of the turbulence quantities with radial distance. In the inner heliosphere, due to shear driving both the quasi-2D and the wave-like component's energy densities decrease less steeply and normalized cross helicities drop strongly. The latter point follows because, for example, $\sigma_{c, z}=\left(Z_{+}^{2}-Z_{-}^{2}\right) /\left(Z_{+}^{2}+Z_{-}^{2}\right)$, and thus adding 


$$
Z^{2}, W^{2}, V_{A}^{2} /(k m / s)^{2}
$$

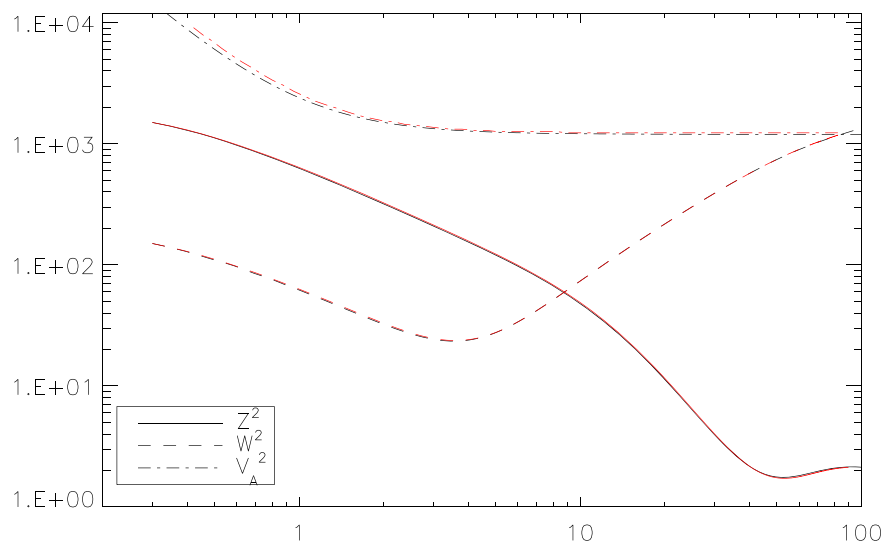

corr. lengths / AU

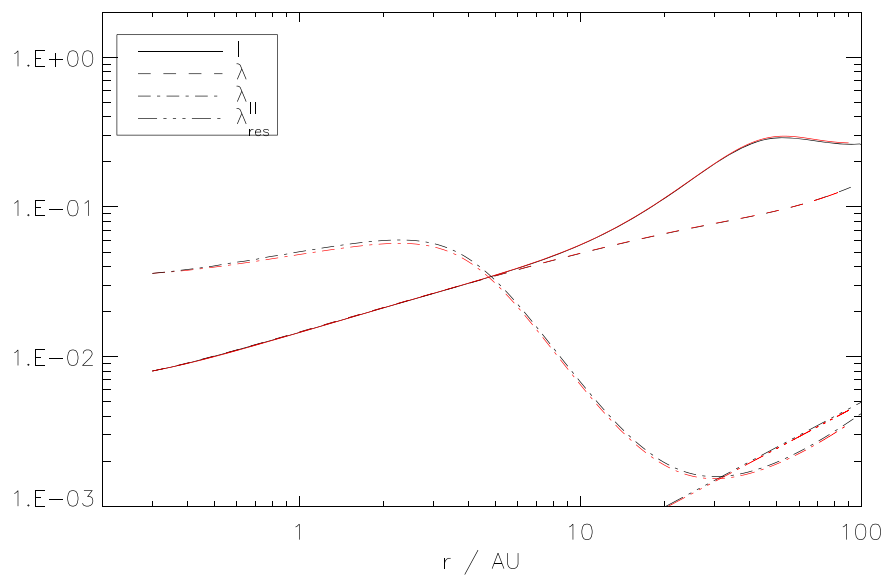

Normalized cross helicities

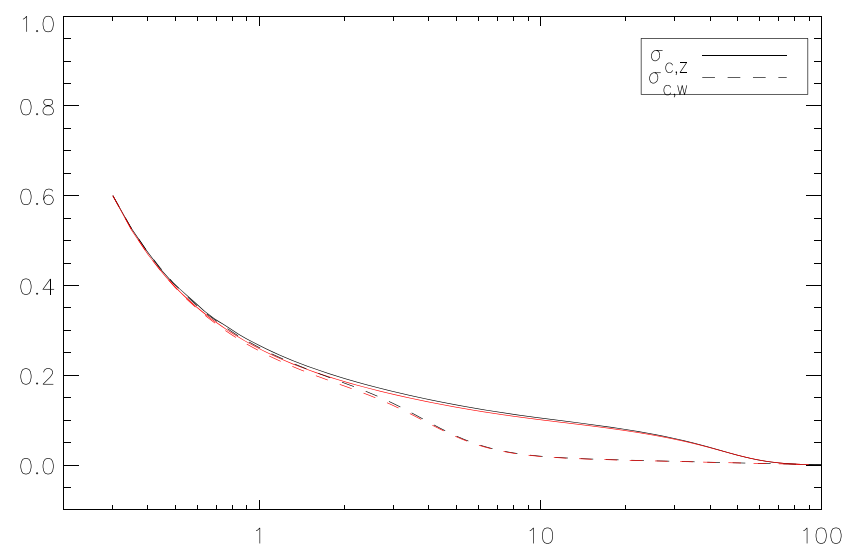

$\mathrm{T} / \mathrm{K}$

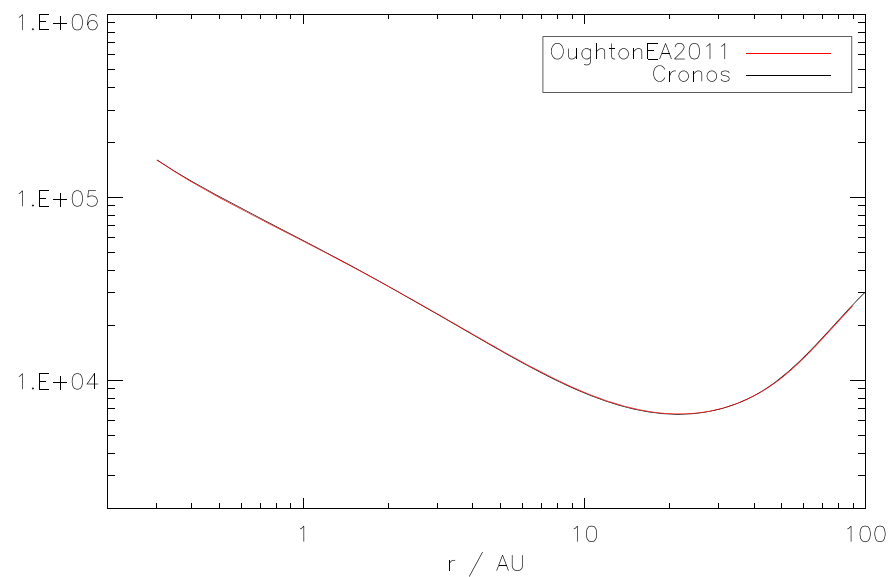

Figure 1. Validation of the CRONOS results (black lines) via a comparison with those obtained previously (red lines) by Oughton et al. (2011) for the Elasser "energies" (upper left panel), the normalized cross helicities (upper right), the correlation lengths (lower left), and the solar wind temperature (lower right).

energy equally to the $Z_{ \pm}^{2}$ leaves the numerator unchanged but increases the denominator (Matthaeus et al. 2004; Breech et al. 2005). As shear driving diminishes with heliospheric distance, the quasi-2D component decays freely while pickup-ion driving feeds only the wave-like component, which, consequently, constitutes the dominant component in the outer heliosphere with its normalized cross helicity $\sigma_{c, w}$ quickly going to zero and its correlation length $\lambda$ much shorter than its quasi-2D counterpart $l$. In the case shown, the pickup driving is strong enough to induce noticable transfer of energy from $W^{2}$ to $Z^{2}$ beyond $\sim 40$ au. This occurs via the "exchange" term, $X^{+}$, in Equations (2) and (3), as discussed in Oughton et al. (2011). There is also an associated decrease of $\ell$ at these distances. Note that the "anti-correlated" behavior of $Z^{2}$ and $\ell$ with heliocentric distance does not hold for $W$ and $\lambda$, which is a consequence of the pickup-ion driving. Furthermore, convergence of the parallel lengthscale toward the pickup-ion gyroradius is also evident. The decaying turbulent energy is dissipated and heats the outer heliosphere as can be seen in the temperature panel. Results obtained with CRONOS (black lines) are shown alongside those obtained with the IDL code (red lines) used in Oughton et al. (2011). An implementation mistake that was present in the latter has since been corrected. The agreement validates the implementation in CRONOS.

\subsection{Extended Model}

The model presented in Section 2, and employed in the remainder of the paper, extends that by Oughton et al. (2011) of the previous section in two ways. First, the background solar wind is no longer prescribed, but computed self-consistently and in a fully three-dimensional manner alongside the turbulence transport equations. Second, the latter are augmented in several ways, namely by (1) not neglecting transport and mixing terms involving the Alfvén velocity, (2) improving the stream shear driving so that it is computed from the background wind, and (3) employing the theory from Isenberg (2005) for the efficiency of pickup-ion driving.

In consequence, the implemented model is applicable to arbitrary solar wind conditions, including sub-Alfvénic heliospheric regions such as the corona and the heliosheath. Coronal models and global heliospheric simulations are both challenging in regard to computer resources, due to the high space and time resolutions required for the former and the long propagation times needed for the latter, especially when including multi-fluid aspects and magnetic fields (e.g., Scherer et al. 2016). We leave such applications for future studies and consider here the super-Alfvénic solar wind during typical solar minimum conditions of fast polar winds and a band of slow wind occupying equatorial regions. We impose azimuthal symmetry, 

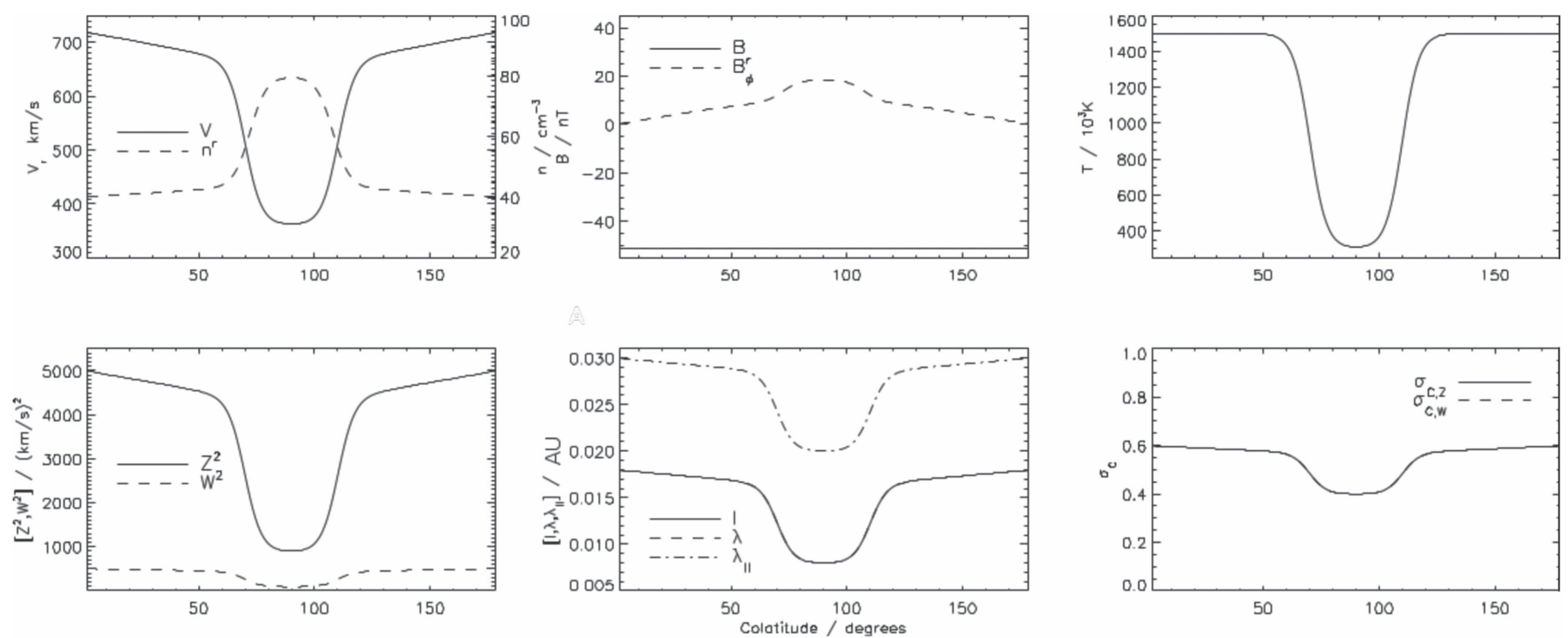

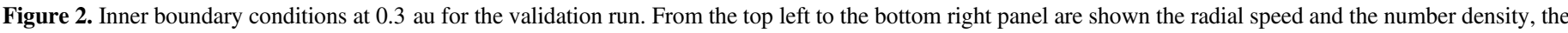

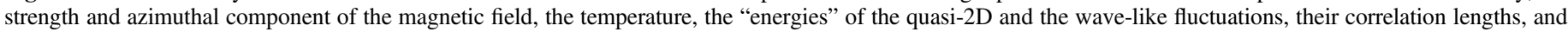
their cross helicities.

which allows for a considerable reduction of computational costs and thereby enables coverage of the full polar angle with one degree resolution. The radial grid is the same as in the previous section, covering the distance from 0.3 to $100 \mathrm{au}$. Figure 2 displays the applied inner boundary conditions depending on colatitude. The top row shows the background quantities (velocity, number density, magnetic field strength, and temperature), in setting which we were guided by Ulysses measurements (McComas et al. 2000). This includes a small latitudinal gradient $\left(\approx 1 \mathrm{~km} / \mathrm{s} /{ }^{\circ}\right)$ of solar wind speed in the fast wind regime, constant mass flux, and a Parker spiral magnetic field structure that neglects a polarity reversal and current sheet. The latter would be under-resolved in these non-AMR simulations and would affect the equatorial results more strongly as appropriate. The bottom row shows the turbulence quantities (turbulent energy density, lengthscales and cross helicities). There is considerable spread and uncertainty associated with spacecraft measurements of these quantities (see Figure 5) and boundary values were chosen to give a reasonable fit to the available data, with the $90 \%-10 \%$ partitioning for $Z^{2}-W^{2}$ guided by observationbased studies (e.g., Bieber et al. 1996; Hamilton et al. 2008). Such studies report a range of values but typically find a dominant quasi-2D component; see Oughton et al. (2015) for a recent review.

Turbulence driven by stream shear can be calculated selfconsistently from the background wind in the present setup, as introduced in Wiengarten et al. (2015). However, the influence of corotating interaction regions, present near solar minimum, is not inherently covered in this simplified geometry with azimuthal symmetry. Moreover, we find that if additional shear is not included in the high-speed regions this results in cross helicities that increase with radial distance (see Dobrowolny et al. 1980a, 1980b), which is in contrast to Ulysses measurements (Figure 5). The source of this additional shear can be attributed to so-called microstreams (Neugebauer et al. 1995). In order to model these additional effects, we include ad hoc terms $C_{\text {add }}^{Z, W}$ in the full driving for $Z$ and $W$, so

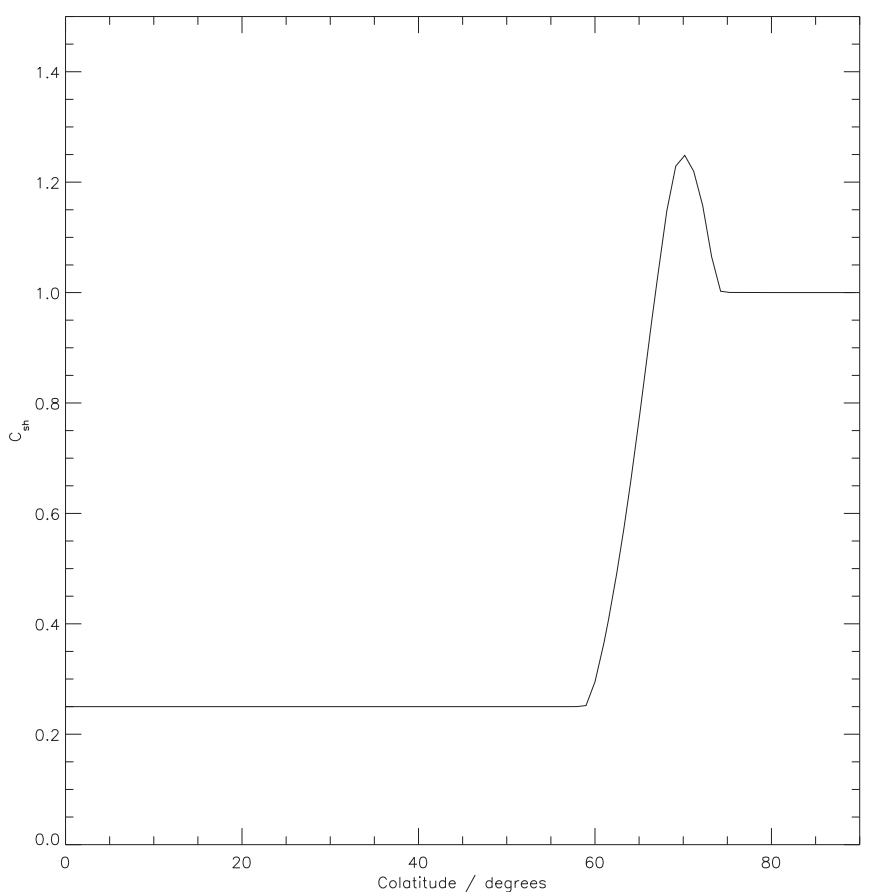

Figure 3. Latitudinal profile of the shear driving term $C_{\mathrm{sh}}$ at 0.5 au according to Equation (22).

that

$$
C_{\mathrm{sh}}^{Z, W}=\frac{1}{|\boldsymbol{U}|}\left(\partial_{\vartheta}+(\sin \vartheta)^{-1} \partial_{\varphi}\right)|\boldsymbol{U}|+C_{\mathrm{add}}^{Z, W},
$$

with $C_{\text {add }}^{Z, W}$ chosen such that in the band of slow wind $C_{\mathrm{sh}}^{Z, W}=1$, while $C_{\mathrm{sh}}^{Z, W}=0.25$ for the fast wind, i.e., a lower bound on shear driving is imposed at all latitudes. The transition region results in higher values and the latitudinal profile of the shear driving displayed in Figure 3 is similar to that used in Breech et al. (2008). 


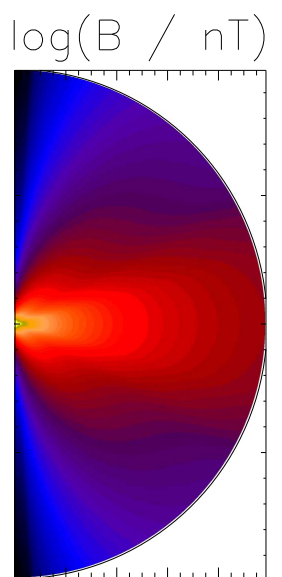

$-020406080$
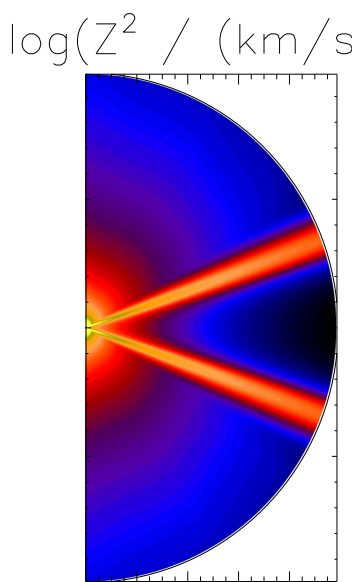

$-020406080$
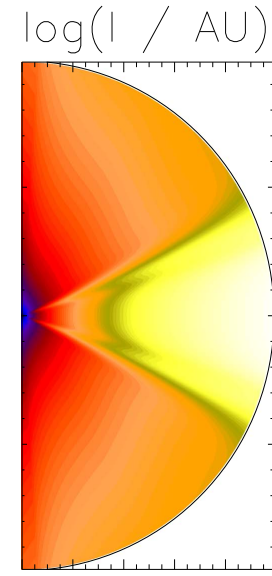

$-020406080$

$\times\lceil A \cup \rrbracket$
$\mathrm{V} / \mathrm{km} / \mathrm{s}$
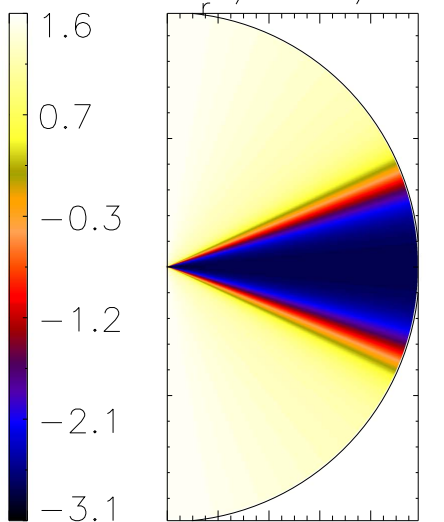

$-020406080$

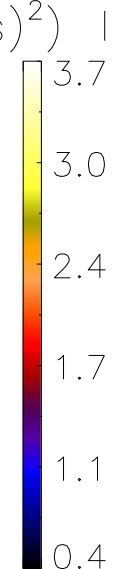

0.4
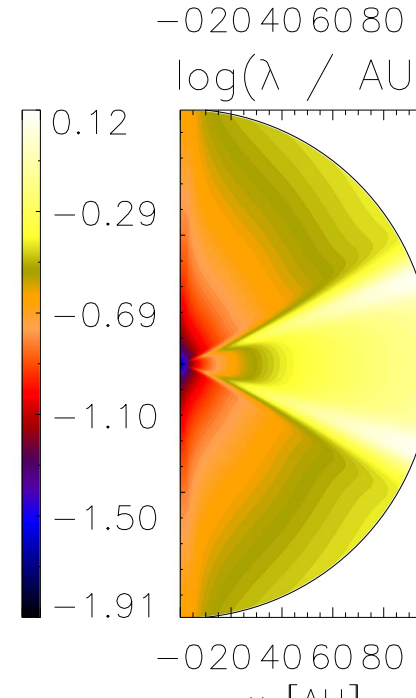

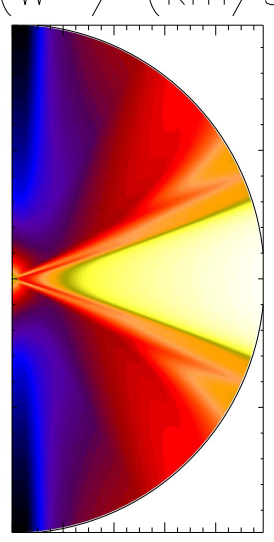

$-020406080$
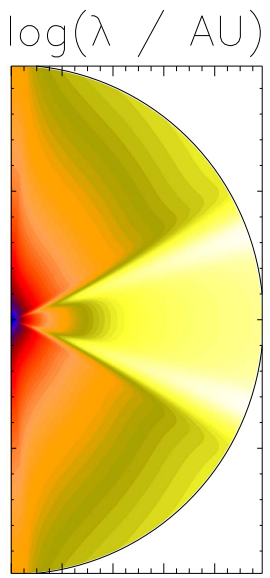

$\times\lceil A \cup\rceil$

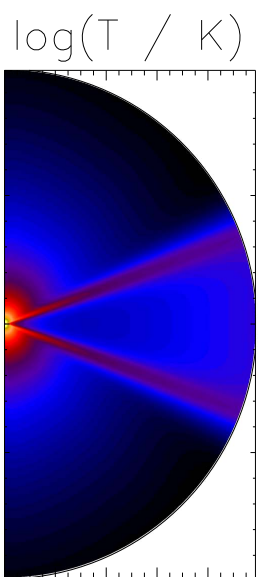

$-020406080$
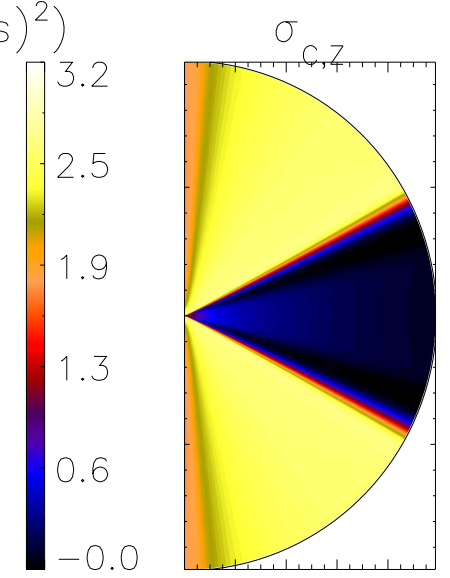

$-020406080$
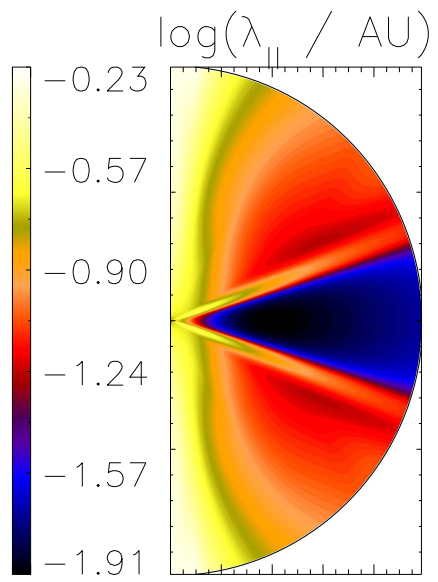

$-020406080$

$\times\lceil A \cup\rceil$

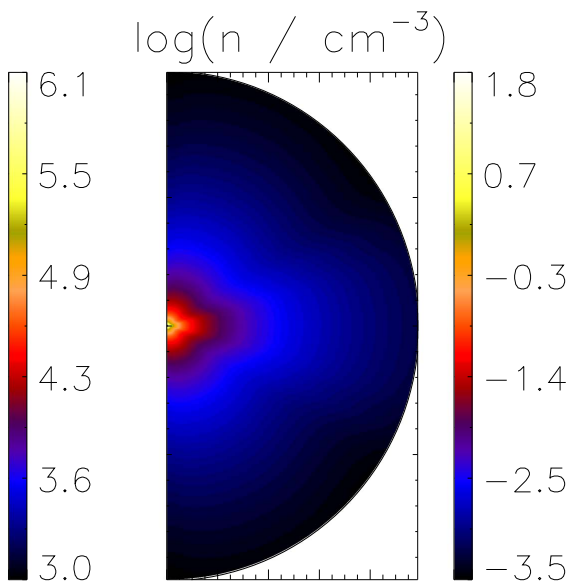

$-020406080$

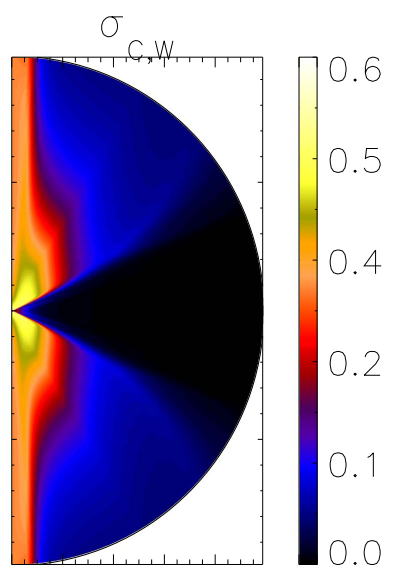

$-020406080$
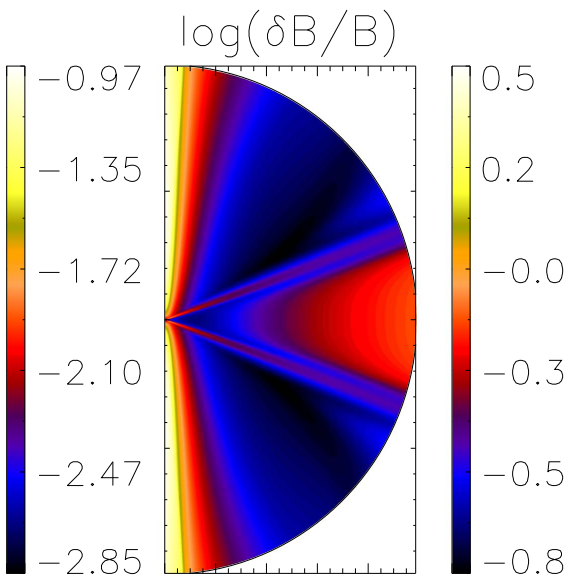

$-020406080$

$\times\lceil A \cup\rceil$

Figure 4. Results of the self-consistent two-component turbulence modeling: contour plots of the background solar wind (top row) and the turbulence quantities (middle and bottom rows) in meridional planes.

The other source for driving turbulence is the excitation of waves via the near isotropization of pickup-ion distributions $\left(\dot{E}_{\mathrm{PI}}\right)$, which we use here in the same form as in Equation (21), but with the efficiency factor $\zeta\left(V_{\mathrm{A}} / U, Z / V_{\mathrm{A}}\right)$ calculated using the improved formulation developed in Isenberg et al. (2003), see also Isenberg (2005).

As before, the residual energy densities are assumed constant with $\sigma_{D}^{z}=\sigma_{D}^{w}=-1 / 3$, and the Kármán-Taylor constants are taken to be $\alpha_{z}=\alpha_{w}=2 \beta_{z}=2 \beta_{w}=0.2$ (Breech et al. 2008; Oughton et al. 2011). The low-latitude inner boundary values at $r_{0}=0.3 \mathrm{au} \quad$ are $Z^{2}=900 \mathrm{~km}^{2} \mathrm{~s}^{-2}, \quad W^{2}=90 \mathrm{~km}^{2} \mathrm{~s}^{-2}$, $\sigma_{c, z}=\sigma_{c, w}=0.4, \quad l=\lambda=0.012 \mathrm{au}, \quad \lambda_{\|}=0.03 \mathrm{au}$, and $T=3.0 \times 10^{5} \mathrm{~K}$, while at high latitudes these values are $Z^{2}=5000 \mathrm{~km}^{2} \mathrm{~s}^{-2}, W^{2}=500 \mathrm{~km}^{2} \mathrm{~s}^{-2}, \sigma_{c, z}=\sigma_{c, w}=0.6$, $l=\lambda=0.018 \mathrm{au}, \lambda_{\|}=0.03 \mathrm{au}$, and $T=1.5 \times 10^{6} \mathrm{~K}$. Simulations are performed until a steady state is reached, for which 

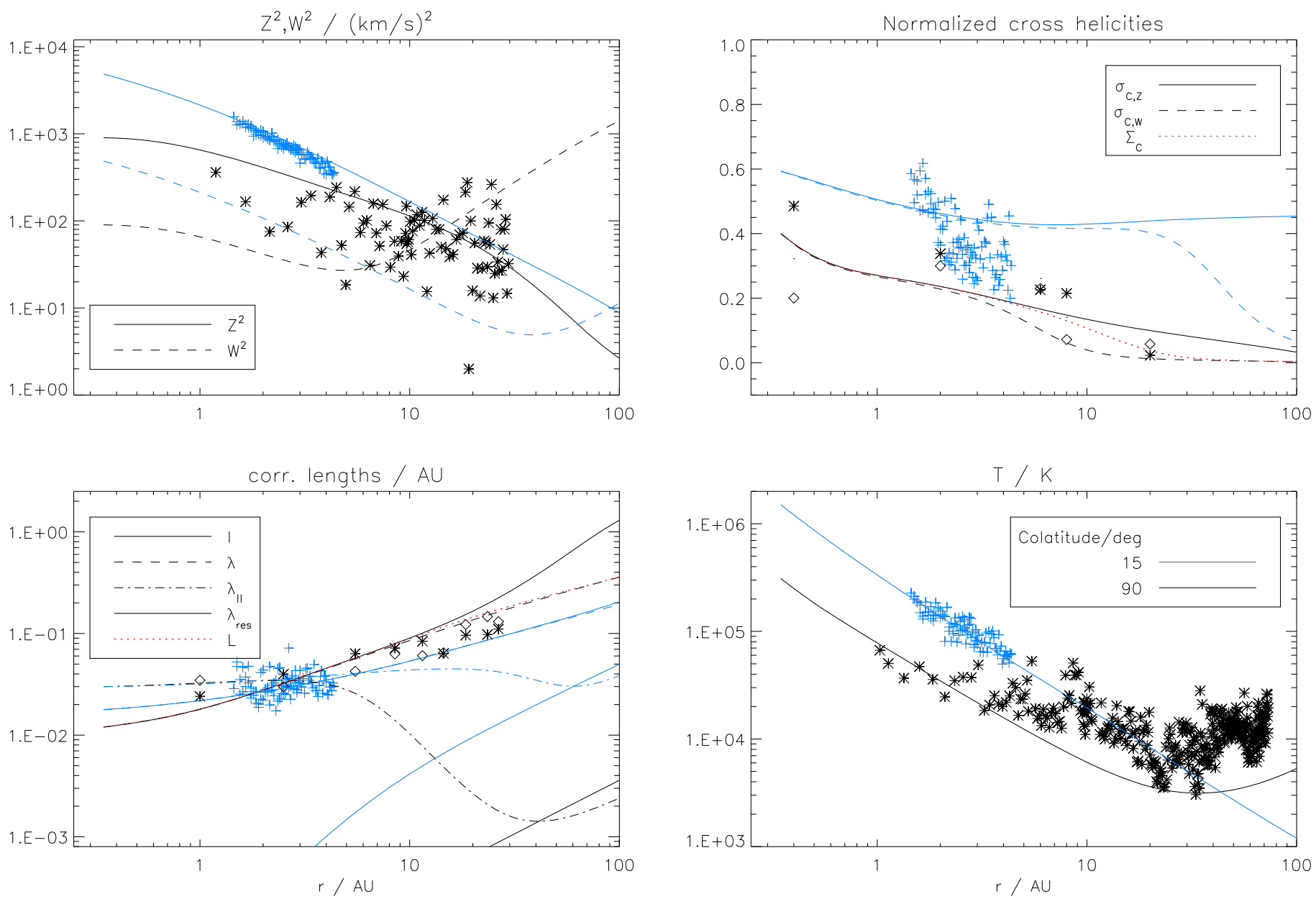

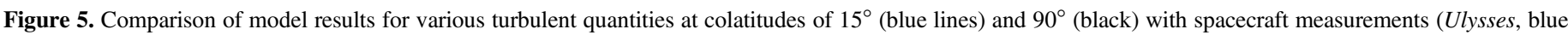

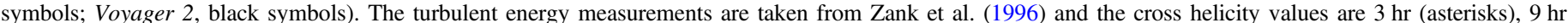

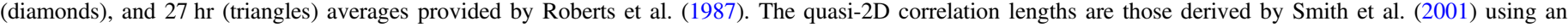
integration (asterisk) and $e$-folding method (diamond). The observed temperature data are also from the latter paper.

the required physical time corresponds approximately to the propagation time from the inner to the outer radial boundary, i.e., about one year. The resulting configuration of the background wind is illustrated in the top row of Figure 4, along with the turbulence quantities in the middle and bottom rows, by contour plots of two-dimensional meridional slices.

The magnetic field exhibits the typical Parker spiral behavior of decreasing more slowly in the ecliptic $\left(\propto r^{-1}\right)$ than above the poles $\left(\propto r^{-2}\right)$, resulting in a constant Alfvén speed in the former and a radially decreasing one in the latter region. The solar wind speed is approximately constant along radial spokes. The background solar wind quantities are barely affected by the inclusion of a turbulence description (Wiengarten et al. 2015), except for some additional heating, mainly occurring in the fast wind/slow wind transition region due to the strong shear there, and in the outer heliosphere due to increased pickup-ion production. The latter effect essentially only acts in the ecliptic plane, because the efficiency factor $\zeta$ tends to zero for small $V_{\mathrm{A}} / U$, as is the case away from the ecliptic plane. This is seen best in the panel for the wave-like turbulence component, $W^{2}$. Also visible are the stripes of enhanced turbulence levels in the transition region, and these are even clearer in the $Z^{2}$ panel.

The regions with stronger generation of turbulence are associated with cross helicities quickly going to zero in their respective component. In other regions, cross helicities that are not equal to zero are retained also at large radial distances, which is not only due to the absence of sources for turbulence, but also because of the inclusion of the additional Alfvén velocity related transport terms, as already demonstrated in Wiengarten et al. (2015) for a one-component turbulence model. Furthermore, the perpendicular lengthscales increase with radial distance as turbulence decays, while the parallel lengthscale approaches the resonant one $\left(\lambda_{\text {res }}\right)$, which is inversely proportional to the magnetic field strength.

Figure 5 shows comparisons of the model results at selected colatitudes with spacecraft measurements. For the fast wind regions, we use Ulysses measurements during its first fast latitude scan (Bavassano et al. 2000a, 2000b, blue crosses) picking out latitudes higher than $35^{\circ}$. Although there is a mixed latitudinal and radial dependence in these data, we use it for comparison with radial dependence of the model data only and choose a colatitude of $15^{\circ}$ (blue lines). Model output in the equatorial plane (black lines) is compared with measurements from the Voyager 2 spacecraft that have been used in previous studies (Roberts et al. 1987; Zank et al. 1996; Smith et al. 2001).

Consider first the high-latitude results. The Ulysses measurements for the turbulent energies (assumed to reside mainly in the quasi-2D component) and temperature show little scattering and are well reproduced by the model, whereas spread in the 
data is large for the correlation lengths and cross helicity. However, the model results are well within the covered range. In the outer heliosphere, pickup-ion driving is evident in $W^{2}$ and $\sigma_{c, w}$ at $r \gtrsim 20 \mathrm{au}$, but only becomes significant in terms of the total fluctuation energy for $r \gtrsim 80 \mathrm{au}$. Since shear driving is also weak in the outer heliosphere, $\sigma_{c, z}$ remains significantly non-zero and there is no strong heating at these high latitudes. This is in contrast to the situation near the ecliptic.

At low latitudes, shear driving is relatively strong inside $\approx 5 \mathrm{au}$, so the radial profiles of the turbulent energies are flatter than their high-latitude counterparts. Pickup-ion driving also becomes important closer in (around $5 \mathrm{au}$ ) and causes the wavelike component to become the dominant one for $r \gtrsim 10 \mathrm{au}$. This leads to a stronger cascade of fluctuation energy and the associated dissipation yields the increasing temperature profile in the outer heliosphere. Thus, it appears that an important reason for the stronger heating near the ecliptic, compared to high latitudes, is the greater radial range where pickup-ion forcing is effective. Voyager measurements show considerable spread but there is again some agreement with the (ecliptic) model results. In particular, the model temperature is a rough lower bound to the observational data and the energy-weighted lengthscale, $L=\left(\ell Z^{2}+\lambda W^{2}\right) /\left(Z^{2}+W^{2}\right)$, passes close to most of the ecliptic data values. Recall that here (and in Wiengarten et al. 2015), Alfvén velocity terms are retained in the transport equations. As Wiengarten et al. (2015) note, this is associated with a shallower radial decrease of $\sigma_{c, z}$ and $\sigma_{c, w}$, compared to transport models, which neglect terms of order $V_{\mathrm{A}} / U$. Moreover, this leads to better agreement with observational data, particularly for the energy-weighted cross helicity $\Sigma_{c}=\left(Z^{2} \sigma_{c, z}+W^{2} \sigma_{c, w}\right) /\left(Z^{2}+W^{2}\right)$, depicted using a red dotted line in Figure 5.

\section{RELEVANCE FOR COSMIC-RAY TRANSPORT COEFFICIENTS}

As mentioned in the Introduction, turbulence transport models such as that presented here are a vital component in ab initio cosmic-ray modulation studies. These models provide information as to the spatial variations of turbulence quantities that feed directly into the diffusion and drift coefficients employed in such modulation studies. Given the relative paucity of in situ spacecraft observations of turbulence in the outer heliosphere, and the extreme sensitivity of computed cosmic-ray intensities to changes in their transport coefficients (see, e.g., Engelbrecht \& Burger 2013, 2015a), a brief outline of the effects of the outputs of a novel turbulence transport model will be of interest to the modulation community. To this end, we present here results for the rigidity and spatial dependences of the proton parallel and perpendicular mean-free paths using outputs yielded by the new, generalized, self-consistent two-component turbulence transport model discussed above. The parallel mean-free path used here is that employed by, e.g., Burger et al. (2008), and derives from quasilinear theory (QLT). We present a novel expression for the proton perpendicular mean-free path, derived from the random ballistic decorrelation (RBD) interpretation of the nonlinear guiding center (NLGC) theory of Matthaeus et al. (2003) as presented by Ruffolo et al. (2012).

The perpendicular mean-free path expressions derived from the NLGC theory or variations on its theme such as the extended NLGC and unified nonlinear theories (see Shalchi 2006, 2010) have already been used in modulation studies. Since these expressions involve, in general, implicit functions, they either need to be evaluated numerically or approximated in some way. The RBD theory has the distinct advantage of yielding explicit expressions for $\lambda_{\perp}$, thereby potentially saving computational time. This, coupled with the fact that the RBD theory provides results in good agreement with numerical simulations, motivates the choice of this scattering theory for the present study.

Assuming axisymmetric fluctuations and a correction for the backtracking of particles, Ruffolo et al. (2012) find that the perpendicular diffusion coefficient can be calculated from the modal spectrum of the 2D magnetic fluctuations $S^{2 \mathrm{D}}$ using

$$
\kappa_{\perp}=\frac{a^{2} v^{2}}{3 B^{2}} \sqrt{\frac{\pi}{2}} \int \frac{S^{2 \mathrm{D}}\left(k_{x}, k_{y}\right)}{k_{\perp} \sqrt{\left\langle\tilde{v}_{x}^{2}\right\rangle}} \operatorname{erfc}(\alpha) \mathrm{d} k_{x} \mathrm{~d} k_{y},
$$

where $k_{\perp}^{2}=k_{x}^{2}+k_{y}^{2}$, and

$$
\alpha=\frac{v^{2} / 3 \kappa_{z z}+\gamma(\boldsymbol{k})}{k_{\perp} \sqrt{2\left\langle\tilde{v}_{x}^{2}\right\rangle}},
$$

with $\kappa_{z z}=v \lambda_{\text {par }} / 3$ the diffusion coefficient parallel to the large-scale field $\boldsymbol{B}$, the particle speed $v$, and the parallel meanfree path $\lambda_{\text {par }}$ of a particle (the latter not to be confused with the correlation scale $\lambda_{\|}$as denoted above). $\gamma(\boldsymbol{k})$ is a damping function that, however, vanishes for the magnetostatic fluctuations assumed here, i.e., $\gamma(\boldsymbol{k})=0$. The quantity $a^{2}$ is a constant, set at a value of $1 / 3$ following Matthaeus et al. (2003), while $B=|\boldsymbol{B}(\boldsymbol{r})|$ denotes the background magnetic field magnitude.

The backtracking-corrected expression is used because, as Ruffolo et al. (2012) show, it provides results in better agreement with simulations. For an isotropic particle velocity distribution, Ruffolo et al. (2012) find that, assuming axisymmetric fluctuations, the average components of the particle guiding center velocity $\tilde{\boldsymbol{v}}$ are given by

$$
\begin{aligned}
& \left\langle\tilde{v}_{x}^{2}\right\rangle=\frac{a^{2} v^{2}}{3} \frac{\delta B_{x}^{2}}{B^{2}}=\left\langle\tilde{v}_{y}^{2}\right\rangle, \\
& \left\langle\tilde{v}_{z}^{2}\right\rangle=\frac{v^{2}}{3}-\left\langle\tilde{v}_{x}^{2}\right\rangle-\left\langle\tilde{v}_{y}^{2}\right\rangle=\frac{v^{2}}{3}\left(1-a^{2} \frac{\delta B^{2}}{B^{2}}\right),
\end{aligned}
$$

with the total variance $\delta B^{2}$ being the sum of the slab and 2D variances, denoted by $\delta B_{2 \mathrm{D}}^{2}$ and $\delta B_{\mathrm{sl}}^{2}$, respectively. Note that, in line with an assumption of axisymmetry, $\delta B_{x}^{2}=\delta B_{2 \mathrm{D}, x}^{2}+\delta B_{\mathrm{sl}, x}^{2}=\left(\delta B_{2 \mathrm{D}}^{2}+\delta B_{\mathrm{sl}}^{2}\right) / 2=\delta B^{2} / 2$, the same holding for $\delta B_{y}^{2}$.

To derive an explicit expression for the perpendicular diffusion coefficient $\kappa_{\perp}$, we employ an expression for the 2D modal spectrum used by Engelbrecht \& Burger (2013):

$$
S^{2 \mathrm{D}}\left(k_{\perp}\right)=g_{0}\left\{\begin{array}{cl}
\left(\lambda_{\text {out }} k_{\perp}\right)^{q}, & \left|k_{\perp}\right|<\lambda_{\text {out }}^{-1} ; \\
1, & \lambda_{\text {out }}^{-1} \leqslant\left|k_{\perp}\right|<\lambda_{2 \mathrm{D}}^{-1} ; \\
\left(\lambda_{2 \mathrm{D}} k_{\perp}\right)^{-\nu}, & \left|k_{\perp}\right| \geqslant \lambda_{2 \mathrm{D}}^{-1} .
\end{array}\right.
$$

where $g_{0}=\left(C_{0} \lambda_{2 \mathrm{D}} \delta B_{2 \mathrm{D}}^{2}\right) /\left(2 \pi k_{\perp}\right)$, and

$$
C_{0}=\left[1-\frac{q}{1+q}\left(\frac{\lambda_{2 \mathrm{D}}}{\lambda_{\text {out }}}\right)+\frac{1}{\nu-1}\right]^{-1}
$$

with $\lambda_{2 \mathrm{D}}$ and $\lambda_{\text {out }}$ lengthscales at which the inertial and energycontaining ranges, respectively, commence. This spectrum has three ranges: an inertial range, an energy-containing range, and 
an "inner" range that decreases as a function of wavenumber. This last range is included due to physical and theoretical considerations, discussed in detail by Matthaeus et al. (2007). In this study, the inertial range spectral index is assumed to equal the Kolmogorov value, so that $\nu=5 / 3$, and the inner range spectral index is set to $q=3$ (see, e.g., Matthaeus et al. 2007). This leads, due to the piecewise definition of Equation (26), to an expression for the perpendicular mean-free path of the form

$$
\lambda_{\perp}=\frac{C_{0} \lambda_{2 \mathrm{D}} \delta B_{2 \mathrm{D}}^{2}}{B \epsilon \lambda_{\|}}\left[h_{\perp, 1}+h_{\perp, 2}+h_{\perp, 3}\right],
$$

where

$$
\begin{aligned}
h_{\perp, 1}= & \frac{1}{q}\left[a \sqrt{3 \pi \epsilon} \lambda_{\text {par }} \operatorname{erfc}\left(x_{1}\right)-3 B \lambda_{\text {out }} E_{(q+1) / 2}\left(x_{1}^{2}\right)\right], \\
h_{\perp, 2}= & 6 B\left(\lambda_{2 \mathrm{D}} x_{2}-\lambda_{\text {out }} x_{3}\right)+a \sqrt{3 \pi \epsilon} \lambda_{\text {par }} \log \left(\frac{\lambda_{\text {out }}}{\lambda_{2 \mathrm{D}}}\right), \\
h_{\perp, 3}= & \frac{a \sqrt{3 \pi \epsilon} \lambda_{\mathrm{par}}}{\nu}\left[\frac { x _ { 4 } ^ { - 1 } } { \sqrt { \pi } } \left(\Gamma\left(\frac{\nu+1}{2}\right)\right.\right. \\
& \left.\left.-\Gamma\left(\frac{\nu+1}{2}, x_{4}^{2}\right)\right)+\operatorname{erfc}\left(x_{4}\right)\right],
\end{aligned}
$$

with, for notational convenience

$$
\begin{aligned}
& x_{1}=\frac{\sqrt{3} B \lambda_{\text {out }}}{a \sqrt{\epsilon} \lambda_{\text {par }}} \\
& x_{2}={ }_{2} F_{2}\left(\frac{1}{2}, \frac{1}{2} ; \frac{3}{2}, \frac{3}{2} ;-x_{4}^{2}\right), \\
& x_{3}={ }_{2} F_{2}\left(\frac{1}{2}, \frac{1}{2} ; \frac{3}{2}, \frac{3}{2} ;-x_{1}^{2}\right), \\
& x_{4}=\frac{\sqrt{3} B \lambda_{2 \mathrm{D}}}{a \sqrt{\epsilon} \lambda_{\mathrm{par}}} .
\end{aligned}
$$

Here $\operatorname{erfc}(x)$ is the complementary error function, $E(x)$ denotes the exponential integral function, $\Gamma(x)$ is the Gamma function, $\Gamma(x, y)$ the incomplete Gamma function, and ${ }_{2} F_{2}$ denotes the generalized hypergeometric function. Note that the variable $\epsilon$ denotes half the total transverse variance, from Equation (25), so that $\epsilon=\delta B_{x}^{2}=\delta B^{2} / 2=\left(\delta B_{2 \mathrm{D}}^{2}+\delta B_{\mathrm{sl}}^{2}\right) / 2, \quad$ assuming axisymmetry.

An expression for the parallel mean-free path is required to evaluate Equation (28). To this end, the QLT proton parallel mean-free path adapted by Burger et al. (2008) from the work of Teufel \& Schlickeiser (2003) is employed:

$$
\begin{aligned}
\lambda_{\mathrm{par}} & =\frac{3 s}{\sqrt{\pi}(s-1)} \frac{R^{2}}{k_{m}}\left(\frac{B}{\delta B_{\mathrm{sl}}}\right)^{2} \\
& \times\left[\frac{1}{4 \sqrt{\pi}}+\frac{2 R^{-s}}{\sqrt{\pi}(2-s)(4-s)}\right],
\end{aligned}
$$

where $R=R_{L} k_{m}$, in terms of the maximal proton gyroradius $R_{L}$ and the wavenumber associated with the slab turnover scale so that $k_{m}=1 / \lambda_{\mathrm{sl}}$. The quantity $s$ denotes the absolute value of the inertial range spectral index (also set to the Kolmogorov value), while $\delta B_{\mathrm{sl}}^{2}$ is the slab variance. Note that Equation (29) is derived assuming a wavenumber-independent energy-containing range on the slab fluctuation power spectrum.

It has been long known, both theoretically and as a result of numerical test particle simulations, that turbulence also has a reducing effect on cosmic-ray drift coefficients (see, e.g., Jokipii 1993; Minnie et al. 2007; Tautz \& Shalchi 2012), although the exact form of such a turbulence-reduced drift coefficient is still not properly understood (Engelbrecht \& Burger 2015b). In this study, we consider the effects of the use of the new, generalized two-component turbulence transport model on two forms of the turbulence-reduced drift coefficient proposed by Burger \& Visser (2010) and Tautz \& Shalchi (2012), both being results of fits to numerical simulations of the drift coefficient for various turbulence scenarios.

The drift coefficient proposed by Burger \& Visser (2010) is based on the result derived by Bieber \& Matthaeus (1997):

$$
\kappa_{\mathrm{A}}=\frac{v}{3} R_{L} \frac{\Omega^{2} \tau^{2}}{1+\Omega^{2} \tau^{2}} .
$$

The drift coefficient can be related to a drift lengthscale by $\kappa_{\mathrm{A}}=v \lambda_{\mathrm{A}} / 3$, where $\Omega$ is the particle gyrofrequency, and $\tau$ a decorrelation rate. These authors choose an expression for $\Omega \tau$ in order to yield a drift coefficient in agreement with simulations performed by Minnie et al. (2007), so that

$$
\Omega \tau=\frac{11}{3} \frac{\sqrt{R_{L} / \lambda_{c, s}}}{\left(D_{\perp} / \lambda_{c, s}\right)^{g}},
$$

where $g=0.3 \log \left(R_{L} / \lambda_{c, s}\right)+1.0$, and $\lambda_{c, s}$ the slab correlation scale. The quantity $D_{\perp}$ denotes the fieldline random walk diffusion coefficient, given by Matthaeus et al. (1995)

$$
D_{\perp}=\frac{1}{2}\left(D_{\mathrm{sl}}+\sqrt{D_{\mathrm{sl}}^{2}+4 D_{2 \mathrm{D}}^{2}}\right)
$$

with

$$
\begin{aligned}
D_{\mathrm{sl}} & =\frac{1}{2} \frac{\delta B_{\mathrm{sl}}^{2}}{B^{2}} \lambda_{c, s}, \\
D_{2 \mathrm{D}} & =\frac{\sqrt{\delta B_{2 \mathrm{D}}^{2} / 2}}{B} \lambda_{u} .
\end{aligned}
$$

The quantity $\lambda_{u}$ represents the $2 \mathrm{D}$ ultrascale, which, for the $2 \mathrm{D}$ turbulence spectral form used in this study, is given by Engelbrecht \& Burger (2013)

$$
\lambda_{u}=\left[C_{0} \lambda_{2 \mathrm{D}}\left(\frac{q}{q-1} \lambda_{\mathrm{out}}-\frac{\nu}{1+\nu} \lambda_{2 \mathrm{D}}\right)\right]^{\frac{1}{2}} .
$$

On the other hand, Tautz \& Shalchi (2012) report a fit to their simulations of the drift coefficient of

$$
\kappa_{\mathrm{A}}=\frac{v}{3} R_{L} \frac{1}{1+c_{1}\left(\delta B^{2} / B^{2}\right)^{c_{2}}},
$$

where $c_{1}=1.09 \pm 0.52$ and $c_{2}=0.81 \pm 0.35$. Both of the above expressions for the turbulence-reduced drift coefficient have been employed in modulation studies, yielding different results for galactic cosmic-ray proton intensities at Earth (Engelbrecht \& Burger 2013, 2015b).

To evaluate Equations (28)-(30) and (35), we employed the self-consistent generalized two-component transport model presented above. This is done under the assumption that the 


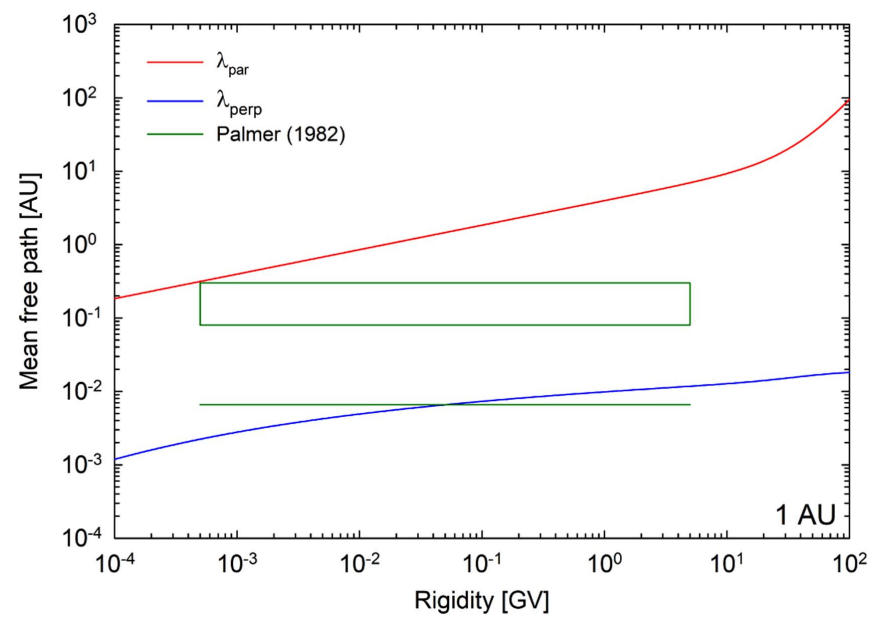

Figure 6. Parallel and perpendicular mean-free paths of Galactic protons as functions of rigidity at 1 au in the ecliptic plane, calculated by using the results of the generalized two-component turbulence transport model. Green box and line denote Palmer (1982) consensus values.

quasi-2D and wave-like quantities provide a reasonable approximation for $2 \mathrm{D}$ and slab quantities, following the approach of Engelbrecht \& Burger (2013), i.e., calculating the variances from

$$
\begin{aligned}
\delta B_{2 \mathrm{D}}^{2} & =\frac{\mu_{0} \rho}{r_{\mathrm{A}}+1} Z^{2}, \\
\delta B_{\mathrm{sl}}^{2} & =\frac{\mu_{0} \rho}{r_{\mathrm{A}}+1} W^{2}
\end{aligned}
$$

where $r_{\mathrm{A}}$ is the Alfvén ratio, assumed to be equal to 0.5 in what follows (see, e.g., Roberts et al. 1987), which corresponds to the value of $\sigma_{D}^{z, w}=-1 / 3$ assumed for the normalized energy difference through the relation $\sigma_{D}^{z, w}=\left(r_{\mathrm{A}}-1\right) /\left(r_{\mathrm{A}}+1\right)$ (e.g., Breech et al. 2008). Furthermore, for the 2D turnover scale $\lambda_{2 \mathrm{D}}$ the weighted quantity $L=\left(Z^{2} l+W^{2} \lambda\right) /\left(Z^{2}+W^{2}\right)$ is used (and shown in the lower left panel in Figure 5), while it is assumed that $\lambda_{\text {out }}=100 \lambda_{2 \mathrm{D}}$. Although perpendicular meanfree paths derived from the NLGC family of scattering theories are quite sensitive to choices made for the 2D outer scale (see, e.g., Engelbrecht \& Burger 2015a), the choice for this quantity is rendered difficult by a lack of observations. Lastly, it should be noted that the normalized cross helicities calculated using the turbulence transport model are not taken into account in the assumed forms of the slab and 2D power spectra used to derive the mean-free paths presented here. This refinement of the modeling will be the subject of future work.

Figure 6 shows the parallel and perpendicular mean-free paths at Earth as a function of rigidity, along with the Palmer (1982) consensus ranges for these quantities. The parallel mean-free path (red line) shows two distinct rigidity dependences, shifting from a $P^{1 / 3}$ dependence below $\sim 10 \mathrm{GV}$ to a $P^{2}$ dependence, as expected from QLT for the spectral form assumed here (see, e.g., Bieber et al. 1994). This quantity remains above the Palmer consensus range (green box) for $\lambda_{\text {par }}$, a consequence of using the results of the generalized twocomponent turbulence transport model. This model is set to reproduce both large-scale and turbulent quantities throughout the heliosphere during solar minimum conditions, during which $\lambda_{\|}$has been previously reported to assume higher values than during times of higher solar activity (Chen \& Bieber 1993).
The perpendicular mean-free path (blue line) also remains partly above the corresponding Palmer consensus range for similar reasons, and shows a rigidity dependence that is slightly steeper than that reported for NLGC-type perpendicular meanfree paths at 1 au by, e.g., Shalchi (2009), Pei et al. (2010), and Engelbrecht \& Burger (2015a).

Regarding spatial dependences, Figure 7 shows contour plots of meridional slices of the logarithms of the parallel (left panel) and perpendicular (right panel) mean-free paths presented here, calculated using the results of the generalized two-component turbulence transport model as discussed in Section 3.2. In the ecliptic plane the radial dependence of the parallel mean-free path initially increases with increasing radial distance, but then flattens out due to the pickup-ion contribution to $W^{2}$. Even though a decrease in $\lambda_{\text {par }}$ would be expected here due to the dependence of Equation (29) on $\delta B_{\mathrm{sl}}^{2}$, this is balanced to some degree by an increase of the proton Larmor radius at these radial distances. At higher latitudes, the flattening of the parallel mean-free path commences at larger radial distances and is less obvious than in the ecliptic, due in part to the latitudinal dependence of the extent of the ionization cavity as modeled here (see Section 3.1 and Figure 5), being governed to a greater extent by the higher values of $R_{L}$ and $\lambda_{\|}$. Generally, at the largest radial distances $\lambda_{\text {par }}$ assumes lower values in the ecliptic, where $W^{2}$ and hence $\delta B_{\mathrm{sl}}^{2}$ are high, than over the poles, where the converse is true for $W^{2}$. Within about 10 au the parallel mean-free path assumes relatively uniform values as function of latitude. This behavior is simply due to the variance.

The perpendicular mean-free path appears to decrease as function of radial distance due to the fact that pickup ions do not directly contribute to $Z^{2}$. This decrease is steeper in the ecliptic plane than at higher latitudes, reflecting the radial decrease in $Z^{2}$ at different latitudes as seen in Figure 5. The perpendicular mean-free path also consistently assumes higher values at higher latitudes than in the ecliptic plane, again a consequence of the behavior of $Z^{2}$, and hence of $\delta B_{2 \mathrm{D}}^{2}$. This dependence also explains the marked increase in $\lambda_{\perp}$ at intermediate latitudes corresponding to regions of enhanced stream-shear effects. Directly above the poles, the perpendicular mean-free path assumes relatively high values, which cannot be associated with a corresponding increase in $Z^{2}$ as seen in Figure 4. This increase can, however, be related to a corresponding increase in the parallel mean-free path, of which $\lambda_{\perp}$ is a function, and to a lesser degree with an increase of the perpendicular correlation scales.

The turbulence-reduced drift scales, calculated from the expressions proposed by Burger \& Visser (2010) and Tautz \& Shalchi (2012; denoted by "BV2010" and "TS2012," respectively), are shown at a rigidity of $1 \mathrm{GV}$ in the left and right panels of Figure 8. Globally, these expressions yield very different results, with the Tautz \& Shalchi (2012) drift scale being, in general, considerably larger than the Burger \& Visser (2010) scale. The latter drift scale displays a considerably more complicated spatial dependence than the former, a consequence of its additional dependences on the various correlation lengthscales calculated in the turbulence transport model. The Burger \& Visser (2010) drift scales become very small at intermediate latitudes due to the enhanced levels of turbulence associated with regions where stream-shear effects are significant. This behavior is not readily apparent when the Tautz \& Shalchi (2012) drift scale is considered. It is 

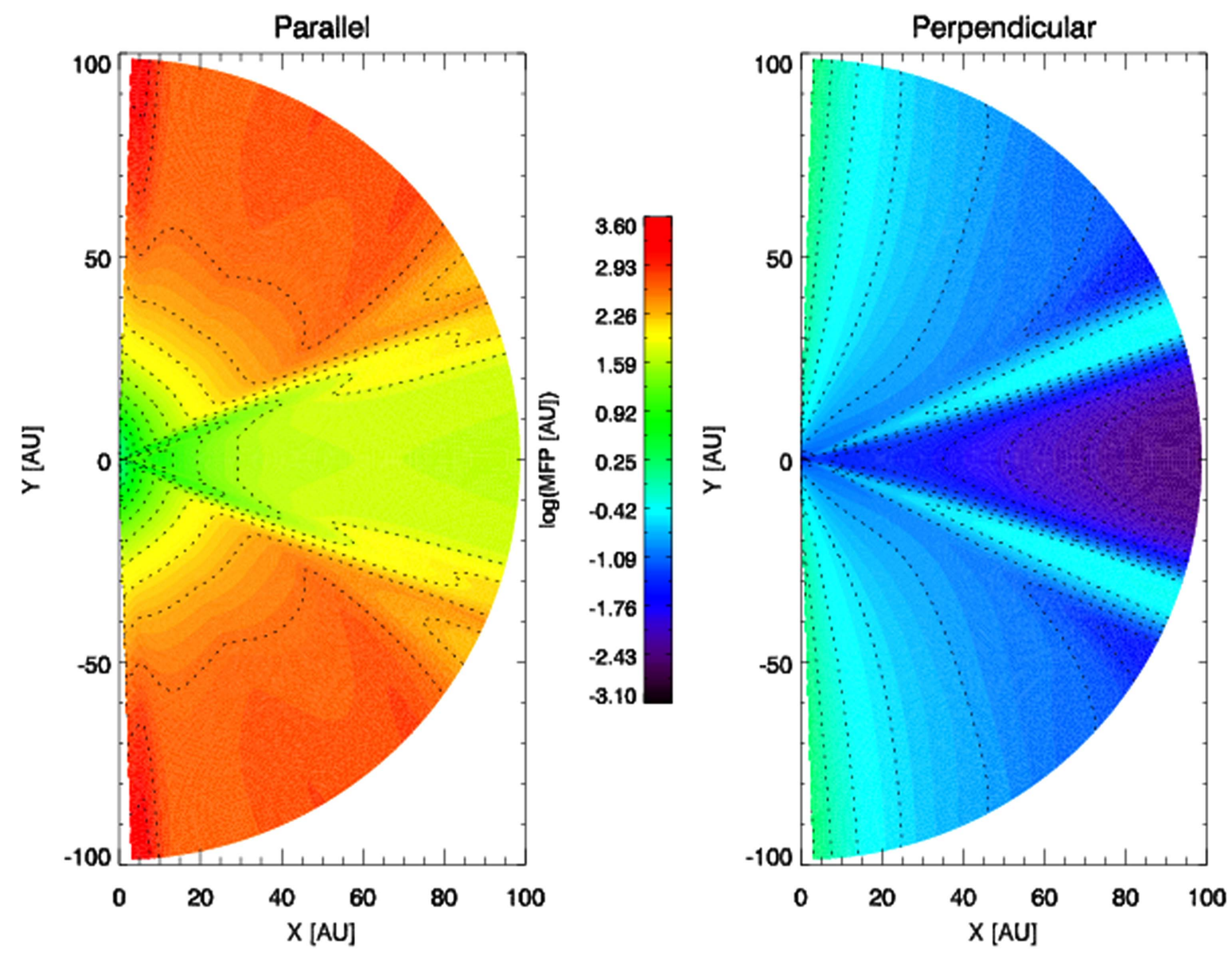

Figure 7. Meridional plane contour plots of the parallel and perpendicular mean-free paths of $1 \mathrm{GV}$ Galactic protons, calculated by using the results of the generalized two-component turbulence transport model.

interesting to note, however, that both drift scales yield results that are larger over the poles than in the ecliptic plane.

The transport coefficients discussed here display complex dependences on the various turbulence quantities, and hence have spatial dependences that are far more complex than those usually assumed in cosmic-ray modulation studies. The latitude dependences of the drift coefficients alone, given the directions in which cosmic rays drift in periods of positive and negative magnetic polarity (see, e.g., Jokipii \& Thomas 1981), can be expected to lead to interesting consequences for modulation studies. Furthermore, given the sensitivity of solutions to the Parker transport equation to choices made for the diffusion and drift terms, the use of self-consistently computed transport coefficients such as those presented here can be expected to lead to new insights in the field of cosmic-ray modulation in both the region enclosed by the termination shock and potentially beyond, i.e., in the inner heliosheath.

\section{SUMMARY AND OUTLOOK}

We have generalized the two-component turbulence model developed by Oughton et al. $(2006,2011)$ to a self-consistent treatment with respect to the solar wind plasma. This generalization consists, first, in a fully three-dimensional formulation of the evolution equations of the two-component phenomenology, i.e., the high-frequency parallel-propagating wave-like and the low-frequency perpendicularly cascading quasi-2D turbulent fluctuations. This includes both a discussion of the most suitable way to formulate the evolution equations for the corresponding correlation lengthscales in order to obtain a closed system for all large-scale and small-scale quantities and a discussion of the correct choice for the structural similarity parameters that implies the occurrence of (in comparison to earlier work, see, e.g., Zank et al. 2012a) additional mixing terms in the equations for the energies (per unit mass) and cross helicities. Second, we have extended the previous modeling by (1) not neglecting transport and mixing terms involving the Alfvén velocity, (2) taking into account the solar wind stream shear, and (3) using a state-of-the-art formulation of the efficiency of the so-called pickup ion driving (Isenberg 2005).

After an implementation in the MHD modeling framework CRONOS (e.g., Wiengarten et al. 2015), the new model, consisting of the generalized turbulence evolution equations self-consistently coupled with those for the large-scale expansion of the solar wind, was validated against the spherically symmetric results obtained earlier by Oughton et al. (2011) for a prescribed background solar wind.

As a first application, we have compared the new threedimensional, self-consistent simulation data with turbulence quantities derived from measurements made with different spacecraft and demonstrated an improvement with respect to earlier models. These improvements comprise the inclusion and improved reproduction of off-ecliptic Ulysses results and, due to the additional Alfvén velocity terms, a better agreement of the computed energy-weighted cross helicity with that derived from observations. 
BV2010

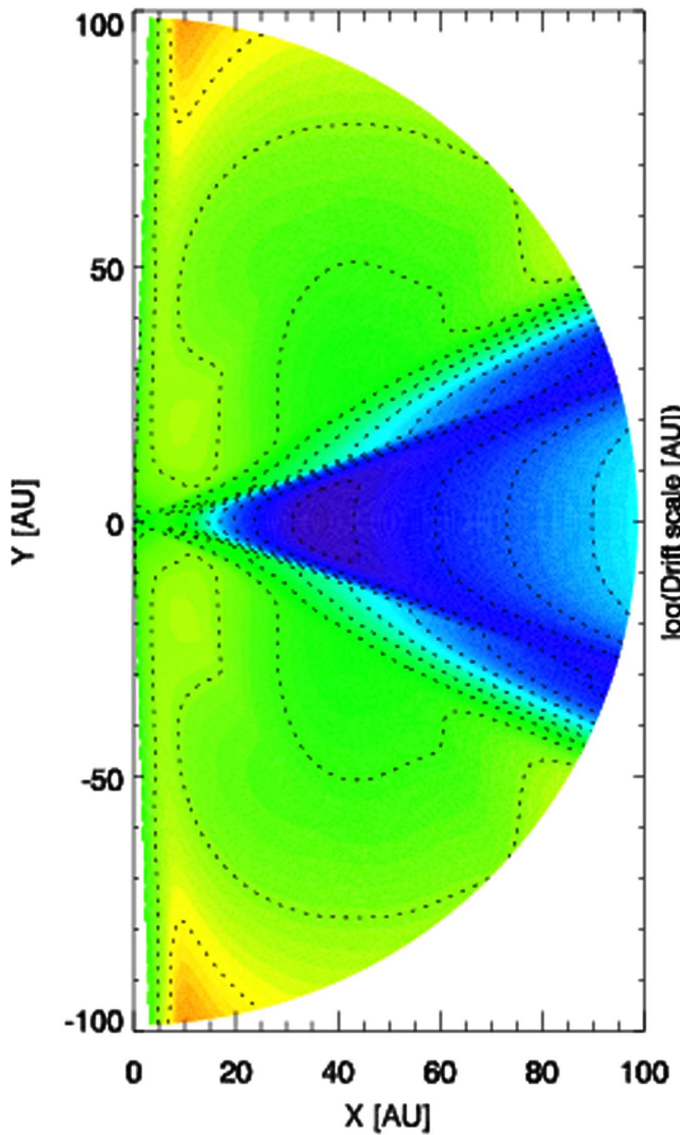

TS2012

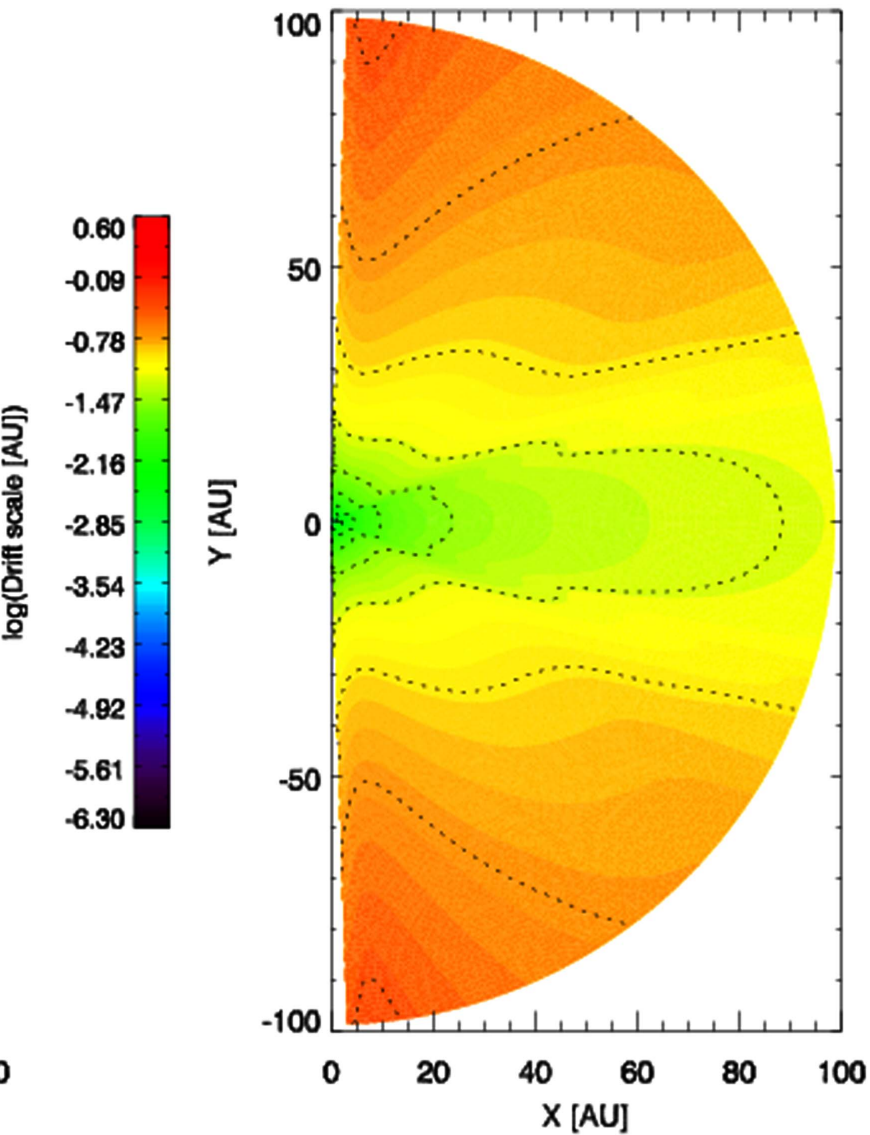

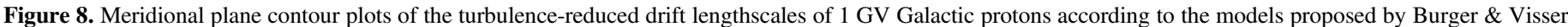
(2010; left panel) and Tautz \& Shalchi (2012; right panel), calculated by using the results of the generalized two-component turbulence transport model.

As a second application, we have used the new results for the wave-like and quasi-2D fluctuations to calculate ab initio diffusion mean-free paths and drifts lengthscales of energetic particles in the turbulent solar wind. Using a well-established result for the quasilinear parallel mean-free path (Teufel \& Schlickeiser 2003; Burger et al. 2008) and a novel expression for the proton perpendicular mean-free path (Ruffolo et al. 2012) derived from the RBD interpretation of the NLGC theory (Matthaeus et al. 2003), we computed values for both quantities that are above the famous Palmer consensus (Palmer 1982; Bieber et al. 1994). Given that the simulations were carried out for solar minimum conditions, this result is in accordance with earlier findings (e.g., Chen \& Bieber 1993). With respect to the particle drifts we employed state-of-the-art expressions derived by Burger \& Visser (2010) and Tautz \& Shalchi (2012) for turbulence-reduced drift scales via fits to simulations of the drift coefficient for various turbulence conditions. While, interestingly, both drift scenarios predict larger scales above the Sun's poles than in the ecliptic plane, they yield rather different results, in general. On the one hand the drift scale of Tautz \& Shalchi (2012) is considerably larger than that of Burger \& Visser (2010). On the other hand, the latter exhibits a comparatively complex spatial dependence as a consequence of its additional dependences on the various correlation lengthscales. In view of the sensitivity of the solution of the cosmic-ray transport equation to the diffusion and drift coefficients, the modeling of their dependence on the underlying turbulence as studied in the present work can be expected to lead to new insights in the field of cosmic-ray modulation, both within and beyond the termination shock.

With the new, generalized two-component model of solar wind turbulence we have demonstrated the feasibility to selfconsistently take into account all terms containing the Alfvén velocity. The explicit incorporation of the latter allowed not only for the extension of the model to all heliographic latitudes and longitudes but will particularly allow quantitative studies of the sub-Alfvénic solar wind regions in the inner heliosphere (as in Wiengarten et al. 2015) close to the Sun and is also a prerequisite for applications to the heliosheath whose turbulent structure is as yet unmodeled.

We thank P. Isenberg for providing his computer code for the calculation of the $\zeta$ parameter in the model for the pickupion driving. N.E.E. thanks D. Ruffolo for many valuable discussions and acknowledges support from the National Research Foundation (Grant 96478). The work benefitted from financial support for T.W. via the DFG project FI 706/14-1 and for H.F., J.K., S.O., and K.S. via the DFG-funded collaboration project FI 706/18-1.

\section{REFERENCES}

Adhikari, L., Zank, G. P., Bruno, R., et al. 2015, ApJ, 805, 63 Bavassano, B., Pietropaolo, E., \& Bruno, R. 2000a, JGR, 105, 12697 Bavassano, B., Pietropaolo, E., \& Bruno, R. 2000b, JGR, 105, 15959 Belcher, J. W., \& Davis, L., Jr. 1971, JGR, 76, 3534 Bieber, J. W., \& Matthaeus, W. H. 1997, ApJ, 485, 655 
Bieber, J. W., Matthaeus, W. H., Smith, C. W., et al. 1994, ApJ, 420, 294 Bieber, J. W., Wanner, W., \& Matthaeus, W. H. 1996, JGR, 101, 2511 Breech, B., Matthaeus, W. H., Minnie, J., et al. 2005, GeoRL, 32, L06103 Breech, B., Matthaeus, W. H., Minnie, J., et al. 2008, JGRA, 113, 8105 Burger, R. A., Krüger, T. P. J., Hitge, M., \& Engelbrecht, N. E. 2008, ApJ, 674,511

Burger, R. A., \& Visser, D. 2010, ApJ, 725, 1366

Chen, J., \& Bieber, J. W. 1993, ApJ, 405, 375

Dobrowolny, M., Mangeney, A., \& Veltri, P. 1980a, PhRvL, 45, 144

Dobrowolny, M., Mangeney, A., \& Veltri, P. 1980b, A\&A, 83, 26

Dosch, A., Adhikari, L., \& Zank, G. P. 2013, in AIP Conf. Ser. 1539, Solar Wind 13, ed. G. P. Zank et al. (Melville, NY: AIP), 155

Engelbrecht, N. E., \& Burger, R. A. 2013, ApJ, 779, 158

Engelbrecht, N. E., \& Burger, R. A. 2015a, ApJ, 814, 152

Engelbrecht, N. E., \& Burger, R. A. 2015b, AdSpR, 55, 390

Grenfell, J. L., Grießmeier, J.-M., von Paris, P., et al. 2012, AsBio, 12, 1109

Grießmeier, J.-M., Tabataba-Vakili, F., Stadelmann, A., Grenfell, J. L., \& Atri, D. 2015, A\&A, 581, A44

Hamilton, K., Smith, C. W., Vasquez, B. J., \& Leamon, R. J. 2008, JGRA, 113 , A01106

Heesen, V., Beck, R., Krause, M., \& Dettmar, R.-J. 2009, A\&A, 494, 563

Hollweg, J. V. 1973, JGR, 78, 3643

Hollweg, J. V. 1974, JGR, 79, 3845

Hollweg, J. V. 1976, JGR, 81, 1649

Iovieno, M., Gallana, L., Fraternale, F., et al. 2016, EJMF, 55, 394

Isenberg, P. A. 2005, ApJ, 623, 502

Isenberg, P. A., Oughton, S., Smith, C. W., \& Matthaeus, W. H. 2010, in AIP Conf. Ser. 1302, Pickup Ions Throughout the Heliosphere and Beyond, ed. J. Le. Roux et al. (Melville, NY: AIP), 180

Isenberg, P. A., Smith, C. W., \& Matthaeus, W. H. 2003, ApJ, 592, 564

Jokipii, J. R. 1993, in Proc. ICRC, 3, 497

Jokipii, J. R., \& Thomas, B. 1981, ApJ, 243, 1115

Klein, L. W., Roberts, D. A., \& Goldstein, M. L. 1991, JGR, 96, 3779

Mao, S. A., Zweibel, E., Fletcher, A., Ott, J., \& Tabatabaei, F. 2015, ApJ, 800,92

Marsch, E., \& Tu, C.-Y. 1990, JGR, 95, 8211

Matthaeus, W. H., Bieber, J. W., Ruffolo, D., Chuychai, P., \& Minnie, J. 2007, ApJ, 667, 956

Matthaeus, W. H., Gray, P. C., Pontius, J. D. H., \& Bieber, J. W. 1995, PhRvL, 75,2136

Matthaeus, W. H., Klein, L. W., Ghosh, S., \& Brown, M. R. 1991, JGR, 96, 5421

Matthaeus, W. H., Minnie, J., Breech, B., et al. 2004, GeoRL, 31, L12803

Matthaeus, W. H., Oughton, S., Pontius, D. H., Jr., \& Zhou, Y. 1994, JGR, 99, 19267

Matthaeus, W. H., Qin, G., Bieber, J. W., \& Zank, G. P. 2003, ApJL, 590, L53

Matthaeus, W. H., Zank, G. P., \& Oughton, S. 1996, JPIPh, 56, 659

Matthaeus, W. H., Zank, G. P., Smith, C. W., \& Oughton, S. 1999, PhRvL, 82,3444
McComas, D. J., Barraclough, B. L., Funsten, H. O., et al. 2000, JGR, 105, 10419

Minnie, J., Bieber, J. W., Matthaeus, W. H., \& Burger, R. A. 2007, ApJ, 670, 1049

Neugebauer, M., Goldstein, B. E., McComas, D. J., Suess, S. T., \& Balogh, A. 1995, JGR, 100, 23389

Oughton, S., Dmitruk, P., \& Matthaeus, W. H. 2006, PhP1, 13, 042306

Oughton, S., Matthaeus, W. H., Smith, C. W., Breech, B., \& Isenberg, P. A. 2011, JGRA, 116, 8105

Oughton, S., Matthaeus, W. H., Wan, M., \& Osman, K. T. 2015, RSPTA, 373 http://rsta.royalsocietypublishing.org/content/373/2041/20140152. full.pdf

Palmer, I. D. 1982, RvGSP, 20, 335

Parker, E. N. 1965, SSRv, 4, 666

Pei, C., Bieber, J. W., Breech, B., et al. 2010, JGRA, 115, 3103

Perri, S., \& Balogh, A. 2010, GeoRL, 37, L17102

Roberts, D. A., Klein, L. W., Goldstein, M. L., \& Matthaeus, W. H. 1987, JGR, 92, 11021

Ruffolo, D., Pianpanit, T., Matthaeus, W. H., \& Chuychai, P. 2012, ApJL, 747, L34

Scalo, J., Kaltenegger, L., Segura, A. G., et al. 2007, AsBio, 7, 85

Scherer, K., Fichtner, H., Kleimann, J., et al. 2016, A\&A, 586, A111

Scherer, K., van der Schyff, A., Bomans, D. J., et al. 2015, A\&A, 576, A97

Schlickeiser, R. 2002, Cosmic Ray Astrophysics, Astronomy and Astrophysics Library (Berlin: Springer)

Shalchi, A. 2006, A\&A, 453, L43

Shalchi, A. 2009, Nonlinear Cosmic Ray Diffusion Theories, Vol. 362 (Berlin: Springer)

Shalchi, A. 2010, ApJL, 720, L127

Smith, W. S., Matthaeus, W. H., Zank, G. P., et al. 2001, JGR, 106, 8253

Snodgrass, H. B., \& Ulrich, R. K. 1990, ApJ, 351, 309

Tautz, R., \& Shalchi, A. 2012, ApJ, 744, 125

Teufel, A., \& Schlickeiser, R. 2003, A\&A, 397, 15

Tu, C.-Y., Pu, Z.-Y., \& Wei, F.-S. 1984, JGR, 89, 9695

Usmanov, A. V., Goldstein, M. L., \& Matthaeus, W. H. 2014, ApJ, 788, 43

Usmanov, A. V., Matthaeus, W. H., Breech, B. A., \& Goldstein, M. L. 2011, ApJ, 727, 84

Wiengarten, T., Fichtner, H., Kleimann, J., \& Kissmann, R. 2015, ApJ, 805,155

Williams, L. L., Hall, D. T., Pauls, H. L., \& Zank, G. P. 1997, ApJ, 476, 366

Zank, G. P. 2014, Transport Processes in Space Physics and Astrophysics, Vol 877 (Berlin: Springer)

Zank, G. P. 2015, ARA\&A, 53, 449

Zank, G. P., Dosch, A., Hunana, P., et al. 2012a, ApJ, 745, 35

Zank, G. P., Jetha, N., Hu, Q., \& Hunana, P. 2012b, ApJ, 756, 21

Zank, G. P., \& Matthaeus, W. H. 1992, JPIPh, 48, 85

Zank, G. P., \& Matthaeus, W. H. 1993, PhFl, 5, 257

Zank, G. P., Matthaeus, W. H., \& Smith, C. W. 1996, JGR, 101, 17093

Zhou, Y., \& Matthaeus, W. H. 1990, JGR, 95, 10291 